\title{
Pin1 has opposite effects on wild-type and P301L tau stability and tauopathy
}

\author{
Jormay Lim,, ${ }^{1}$ Martin Balastik, ${ }^{1}$ Tae Ho Lee, ${ }^{1}$ Kazuhiro Nakamura, ${ }^{1}$ Yih-Cherng Liou, ${ }^{1}$ \\ Anyang Sun, ${ }^{1}$ Greg Finn, ${ }^{1}$ Lucia Pastorino, ${ }^{1}$ Virginia M.-Y. Lee, ${ }^{2}$ and Kun Ping Lu'

\begin{abstract}
${ }^{1}$ Cancer Biology Program, Department of Medicine, Beth Israel Deaconess Medical Center, Harvard Medical School, Boston, Massachusetts, USA. ${ }^{2}$ Center for Neurodegenerative Disease Research, Department of Pathology and Laboratory Medicine,
\end{abstract} \\ University of Pennsylvania School of Medicine, Philadelphia, Pennsylvania, USA.
}

\begin{abstract}
Tau pathology is a hallmark of many neurodegenerative diseases including Alzheimer disease (AD) and frontotemporal dementia with Parkinsonism linked to chromosome 17 (FTDP-17). Genetic tau mutations can cause FTDP-17, and mice overexpressing tau mutants such as P301L tau are used as AD models. However, since no tau mutations are found in $\mathrm{AD}$, it remains unclear how appropriate tau mutant mice are as an $\mathrm{AD}$ model. The prolyl isomerase Pin 1 binds and isomerizes tau and has been implicated in protecting against neurodegeneration, but whether such Pin1 regulation is affected by tau mutations is unknown. Consistent with earlier findings that Pin1 KO induces tauopathy, here we demonstrate that Pin1 knockdown or KO increased WT tau protein stability in vitro and in mice and that Pin 1 overexpression suppressed the tauopathy phenotype in WT tau transgenic mice. Unexpectedly, Pin1 knockdown or KO decreased P301L tau protein stability and abolished its robust tauopathy phenotype in mice. In contrast, Pin 1 overexpression exacerbated the tauopathy phenotype in P301L tau mice. Thus, Pin 1 has opposite effects on the tauopathy phenotype depending on whether the tau is WT or a P301L mutant, indicating the need for disease-specific therapies for tauopathies.
\end{abstract}

\section{Introduction}

Tau-related phenotypes are prominent common features of many neurodegenerative diseases, called tauopathies, that include Alzheimer disease (AD), frontotemporal dementia with Parkinsonism linked to chromosome 17 (FTDP-17), corticobasal degeneration, progressive supranuclear palsy, and Pick disease (reviewed in refs. 1-3). Tau binds microtubules and promotes their polymerization, thereby playing a crucial role in maintaining the physiological function of healthy neurons (4). Phosphorylation is a key regulatory mechanism that disrupts the ability of tau to bind microtubules and to promote their assembly (4). Dynamic tau phosphorylation occurs during embryonic development and cell division and to some extent in postmitotic neurons and may play important roles in refining or maintaining neuronal structure and function (5). However, aberrant tau phosphorylation in mature neurons is believed to be harmful to the neuron (6). Although it has been debated whether tau hyperphosphorylation and aggregation in the neuron are the cause or the effect of the tauopathies, recent identification of tau genetic mutations in FTDP-17 patients has provided evidence that tau dysfunction can cause the tauopathy phenotype (1-3).

While not found in $\mathrm{AD}$, genetic tau mutations have been identified in FTDP-17 patients (1-3). Moreover, Tg mice overexpressing human P301L tau, one of the most frequent FTDP-17 tau mutants, develop the robust tauopathy phenotype and thus are widely used as models of $\mathrm{AD}$ by itself or in combination with $\mathrm{A} \beta$-related mouse models (7-14). There have been various studies comparing WT tau and P301L mutant tau to better understand their differences

Nonstandard abbreviations used: AD, Alzheimer disease; APP, amyloid precursor protein; FTDP-17, frontotemporal dementia with Parkinsonism linked to chromosome 17; KD, knockdown; NFT, neurofibrillary tangles; Pin1, protein interacting with NIMA 1

Conflict of interest: The authors have declared that no conflict of interest exists. Citation for this article: J. Clin. Invest. 118:1877-1889 (2008). doi:10.1172/JCI34308 in binding to microtubules or regulatory enzymes, forming tau filaments or causing neurotoxicity (1-3). However, it is not fully clear what impact these mutations have on tau regulation, and it is unclear how appropriate such tau mutant mice are as AD models.

Characterization of Tg mice overexpressing tau or its mutants reveals a major impact of tau protein levels on tau-related phenotypes (7-9, 15-21). For example, low levels affect tau compartmentalization and its phosphorylation, similar to the pathological changes characteristic of the so-called pretangle stage of AD $(16,17)$. However, high levels can also cause age-dependent neurodegenerative phenotypes $(18,20)$, as occurs in Tg mice overexpressing tau mutants, which are usually expressed at much higher levels $(7,19,22)$. A key factor in the development of tau-related phenotypes appears to be tau phosphorylation, especially on Ser or Thr residues preceding a Pro (Ser/Thr-Pro) by Pro-directed kinases $(23,24)$ or phosphatases such as PP2A $(25,26)$. Indeed, overexpression of GSK3 $\beta$ or CDK5 activator p 25 or inhibition of PP2A enhances or induces tau-related phenotypes in mice (11, 27-30). In addition, tau phosphorylation has been shown to promote tau degradation via chaperone-interacting protein/Hsp70 (31-33) or to inhibit tau degradation (34). These results indicate that tau phosphorylation is intricately regulated, but nothing has been known of whether tau is further regulated between phosphorylation and dephosphorylation until recently.

We have previously shown that specific pSer/Thr-Pro motifs in certain proteins such as tau can exist in 2 distinct cis and trans conformations, whose conversion can be greatly accelerated by a unique prolyl isomerase, Pin 1 (protein interacting with NIMA 1) (35-38). Subsequent studies indicate that such Pin1-catalyzed conformational changes, which can now be visualized by NMR (39), have a profound impact on phosphorylation signaling, achieved by regulating a spectrum of target activities (40-42). Importantly, Pin 1 is tightly regulated and its deregulation contributes to pathological conditions, notably $\mathrm{AD}$ and cancer (39-45). 
In AD, Pin 1 binds to and isomerizes the pThr231-Pro motif in tau to restore its ability to bind microtubules and to promote their assembly (46) as well as to facilitate tau dephosphorylation by PP2A since the phosphatase dephosphorylates only the trans pSer/Thr-Pro motifs $(47,48)$. Pin 1 also binds to and isomerizes the pThr668-Pro motif in amyloid precursor protein (APP) to promote nonamyloidogenic APP processing and to reduce $A \beta$ production (39). Therefore, Pin 1 acts on both tau and APP, 2 key molecules in AD. Importantly, Pin 1 expression is induced during neuronal differentiation and is highly expressed in most neurons in the brain $(46,48,49)$. However, in the AD neuron, Pin 1 is downregulated or inactivated by oxidative modifications, promoter polymorphisms, or other mechanisms (48, 50-52). Importantly, ablation of the Pin1 gene alone (Pin1 KO, $\mathrm{Pin1}^{-(-)}$in mice is sufficient to induce tau- and $\mathrm{A} \beta$-related phenotypes and neurodegeneration in an age-dependent manner $(39,48)$. These results indicate that loss of Pin 1 function contributes to the development of the tauopathy phenotype in $\mathrm{AD}(42)$. However, it remains to be determined whether Pin 1 overexpression can inhibit tau-related phenotypes in AD and whether Pin 1 regulates FTDP-17 tau mutants in the same way as the WT protein. Answers to these questions are important because they may facilitate the development of new therapies for $\mathrm{AD}$ and related disorders.

In this paper, we investigated the role of Pin 1 in the development of tau-related phenotypes induced by WT tau and its P301L tau mutant both in cell cultures and animal models and surprisingly uncovered the diametrically opposite impacts of Pin 1 on tau protein stability and tauopathy phenotype depending on whether the tau is WT or P301L mutant. These results indicate the importance of using relevant animal models for studying AD and FTDP-17 and also suggest that Pin1 upregulation might be beneficial for $\mathrm{AD}$, whereas its inhibition might be helpful for patients carrying the P301L tau mutation.

\section{Results}

Pin1 knockdown in cells affects protein stability of WT tau and P301L tau in opposite manners via acting on tau proteins. Pin 1 binds to and isomerizes the pThr231-Pro motif in tau to regulate tau dephosphorylation and its microtubule-binding activity (46-49). Interestingly, it has been shown that tau phosphorylation regulates its degradation (31-34) and that protein degradation is modulated by Pin 1 (42). These results suggest that Pin 1 might act on the pThr231-Pro motif in WT tau and P301L tau to regulate their protein stability since the P301L mutation does not affect tau binding to Pin1 (46).

To examine this possibility, we used retroviruses or lentiviruses carrying 2 different Pin 1 RNAi constructs to stably deplete Pin 1 in 2 different human cell lines, HT1080 fibrosarcoma cells and SH-SY5Y neuroblastoma cells, as described (53). Both RNAi constructs efficiently depleted Pin 1 protein in both cell lines (Figure 1 and data not shown). To determine whether Pin 1 knockdown (KD) affects protein stability of WT tau and P301L tau, Pin1 KD and control cells were cotransfected with tau or its P301L mutant expression construct overnight, followed by the addition of cycloheximide to inhibit de novo tau synthesis, followed by determination of the steady-state levels of tau at various times, as described $(54,55)$. Under this cycloheximide chase analysis, the rate at which tau steady-state levels decline reflects the half-life of the proteins. In control SY5Y cells and HT1080 cells, WT tau was unstable but became highly stable in both types of Pin $1 \mathrm{KD}$ cells (Figure 1, A and B, and data not shown). In contrast, P301L tau was highly unstable in Pin1 KD cells, as compared with control cells, where it was much stabler (Figure 1, C and $\mathrm{D}$, and data not shown). Importantly, these effects of Pin $1 \mathrm{KD}$ on tau protein stability were completely abolished in the presence of the proteosome inhibitor MG132, where both WT tau and P301L tau were stable and their levels were not affected by Pin1 KD (Figure 1, A-D). These results demonstrate that Pin $1 \mathrm{KD}$ has opposite impacts on protein stability of WT tau and P301L tau both in nonneuronal and neuronal cells and that the impact depends on functional proteosome, as shown for other Pin 1 substrates (42).

To exclude off-target effects of Pin $1 \mathrm{KD}$, we transfected Pin 1 RNAi stable SY5Y cells with a vector expressing the Pin 1 coding sequence because the Pin1 RNAi sequence is directed against the $3^{\prime}$ untranslated region about $600 \mathrm{bp}$ downstream of the stop codon. As expected, the cells reexpressed Pin 1 (Figure 1). Importantly, Pin 1 addback completely rescued the opposite effects of Pin 1 KD on protein stability of WT tau and P301L tau; it rendered WT tau unstable but P301L tau as stable in Pin1 KD cells (Figure 1, A-D). These results indicate that the observed effects on tau protein stability are specifically due to depletion of Pin 1.

To determine whether the effects of Pin 1 KD on tau stability depend on the presence of the Pin1-binding site in tau proteins, we used the Thr231Ala mutation, which abolishes the ability of Pin 1 to act on tau $(46,47)$. Protein stability of both tau-T231A and P301L tau-T231A mutant was affected neither by Pin $1 \mathrm{KD}$ nor by reexpression of Pin 1 in Pin 1 KD cells (Figure 1, E-H). These results indicate that the effects of Pin 1 on both WT tau and P301L depend on the presence of the Pin1-binding site in tau, as shown for other Pin 1 targets (42).

To determine whether Pin1 KO has any effects on protein stability of other FTDP-17 tau mutants, we selected 3 other commonly studied FTDP-17 tau point mutants, P301S, V337M, and R406W (56-58). Similar to P301L tau (Figure 1, C and D, and Figure 2, $\mathrm{A}$ and $\mathrm{B}), \mathrm{P} 301 \mathrm{~S}$ tau was less stable in Pin1 KD cells than in control cells (Figure 2, C and D). In contrast, both V337M tau and R406W tau were more stable in Pin 1 KD cells than in control cells (Figure 2, E-H), as seen in WT tau (Figure 1, A and B). Although further experiments are needed to examine other FTDP-17 tau mutants, these studies suggest that the effects of Pin 1 on FTDP17 tau mutants likely depend on the identity of the tau mutations. Given that P301L tau probably is the most well-studied FTDP tau mutant, especially with widely used Tg mouse models (7-14), we will here focus our study on comparing the impact of Pin 1 on WT tau and P301L tau.

Pin 1 KO affects protein stability of WT tau and P301L tau in primary neurons and in brain tissues in an opposite manner. To confirm the above surprising observations, we examined the effects of Pin $1 \mathrm{KO}$ on protein stability of WT tau and P301L tau using primary neuron cultures derived from Pin 1 WT or KO mice and using organotypic brain-slice cultures derived from bigenic mice generated from crosses between Pin 1 KO mice and WT tau or P301L Tg mice.

To determine the effects of Pin $1 \mathrm{KO}$ on protein stability of WT tau and P301L tau in primary neurons, we established cortical neuron cultures derived from $P i n 1^{+/+}$and Pin1 $1^{-/-}$embryos at day E17.5 and infected them with lentiviruses expressing WT tau or P301L tau, followed by cycloheximide chase analysis to determine their half-lives. To distinguish exogenous tau proteins from the endogenous protein, we inserted an N-terminal His tag to WT tau and P301L tau. As compared with the corresponding control in control Pin1 ${ }^{+/+}$neurons, transfected WT tau was significantly more stable, whereas transfected P301L tau was much less stable in Pin1 $1^{-/-}$neurons (data not shown). These results demonstrate that Pin $1 \mathrm{KO}$ has the opposite effects on protein stability of WT and P301L tau in primary neuron cultures. 
A
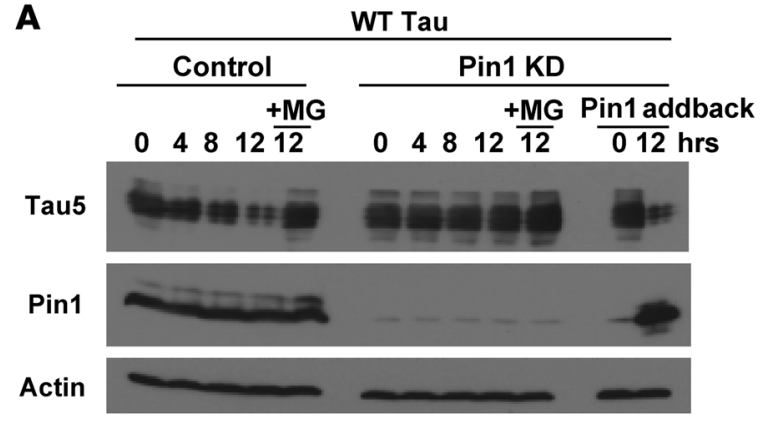

C
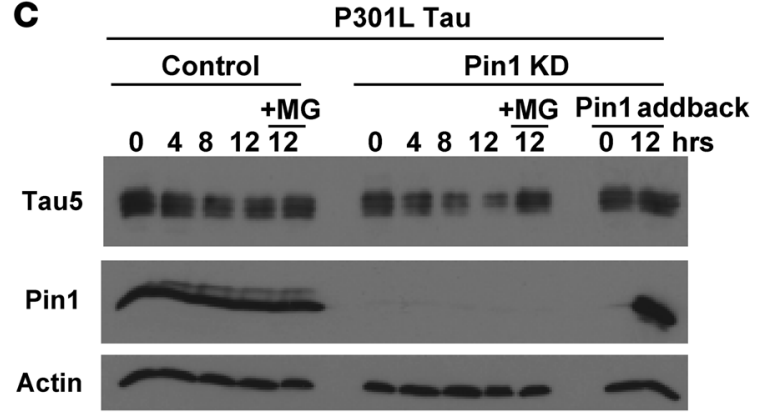

$\mathbf{E}$
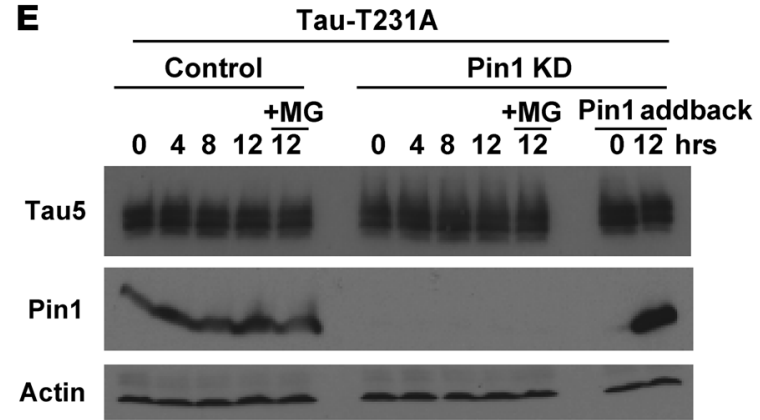

G P301L Tau-T231A
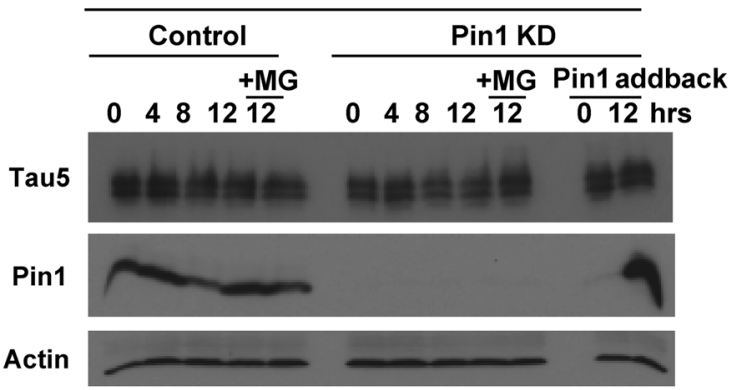

B

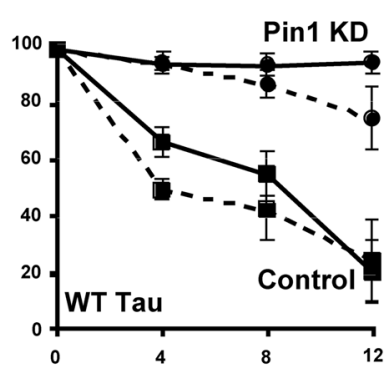

D

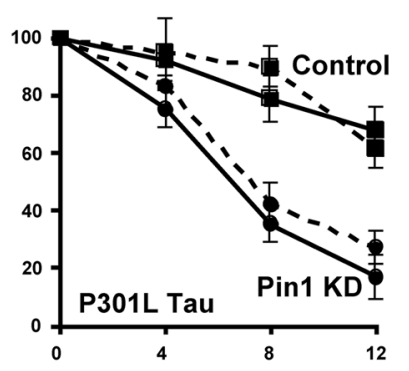

$\mathbf{F}$

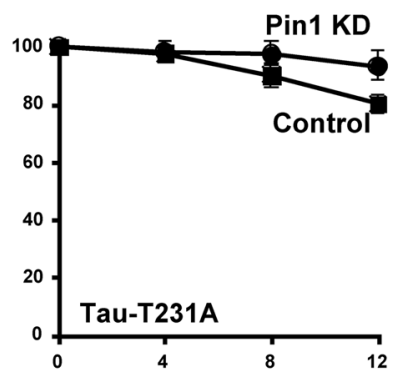

H

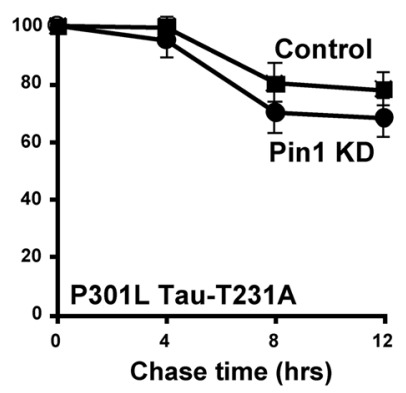

Figure 1

Pin1 KD in cells has opposite effects on the protein stability of WT tau and P301L tau; these effects depend on the presence of the Pin1-binding site in tau proteins. SH-SY5Y cells were infected with Pin1 siRNA or control siRNA lentiviruses, followed by selection for stable cells with puromycin. Stable Pin1 KD or control SY5Y cells were transfected overnight with a WT tau expression construct in the presence or absence of a Pin1 expression construct (+Pin1 addback) (A and B), with a P301L tau construct in the presence or absence of a Pin1 construct (C and D), with a tau-T231A construct in the presence or absence of a Pin1 construct ( $E$ and $\mathbf{F}$ ), or with a P301L tau-T231A construct in the presence or absence of a Pin1 construct ( $\mathbf{G}$ and $\mathbf{H}$ ). Cycloheximide was added to stop new protein synthesis and chased for indicated times in the absence or presence of the proteosome inhibitor MG132. Cells were harvested and cell lysates were fractionated by SDS-PAGE and analyzed by immunoblotting with Tau5, anti-Pin1, or anti-actin antibodies (A, C, E, and $\mathbf{G})$. Tau levels were semiquantified from 3 different experiments using ImageQuant and normalized using actin as an internal control (B, D, F, and H). For comparison, tau levels at 0 (before the cycloheximide addition) were defined as $100 \%$. The opposite effects of Pin1 KD on protein stability of WT tau and P301L tau were also obtained in HT1080 cells, as shown by dashed lines in $\mathbf{B}$ and $\mathbf{D}$. Error bars represent SD.
To examine the effects of Pin1 KO on protein stability of WT tau and P301L tau in brain tissues, we established brain-slice cultures from WT tau or Tg P301L tau mice in the presence or absence of Pin1, followed by cycloheximide chase analysis to examine the half-lives of WT tau and P301L tau in brain tissues. Tg WT tau had a half-life of approximately 12 hours in the presence of Pin 1 but became almost completely stable in the absence of Pin1 (Figure 3 , A and B). In contrast, the half-life of Tg P301L tau was significantly decreased to approximately 6 hours in the absence of Pin 1 from approximately 24 hours in the presence of Pin 1 (Figure 3, $\mathrm{C}$ and D). These results convincingly demonstrate that Pin 1 has opposing effects on protein stability of WT tau and P301L tau even in brain-slice cultures, a model that maintains the 3D architecture and synaptic connections of the brain closely resembling the in vivo situation. Although further experiments are needed to elucidate how Pin1 regulates tau protein stability and how P301L mutation of tau causes Pin 1 regulation to move in the opposite direction, we decided to focus our efforts on determining the significance of this Pin1-dependent regulation, which we believe is novel, in the development of tau-related phenotypes induced by WT tau and P301L tau in vivo because it might have important therapeutic implications for treating human tauopathies.

Pin1 KO abrogates tau-related phenotypes in P301L tau Tg mice. The above findings that Pin $1 \mathrm{KD}$ or $\mathrm{KO}$ in cell cultures and in brain-slice 
A

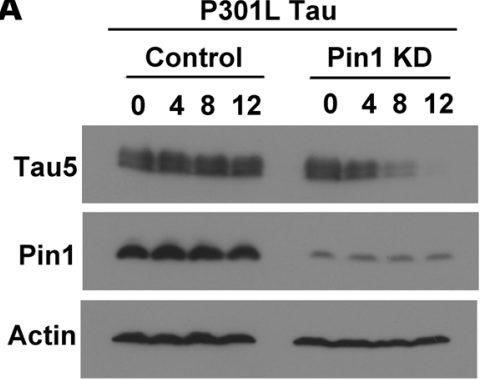

C

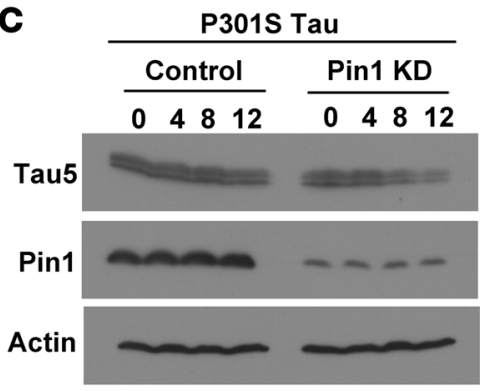

$\mathbf{E}$

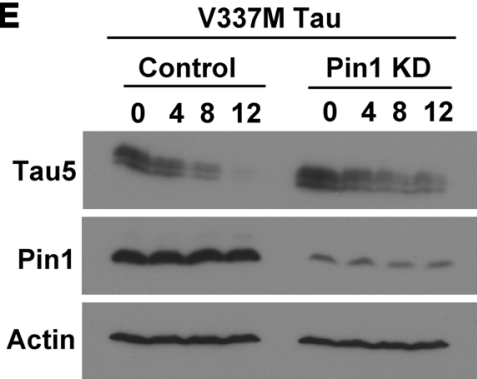

G
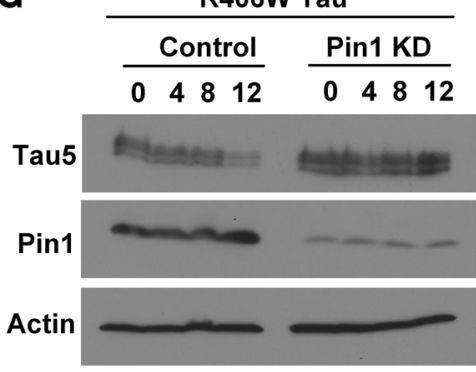

B

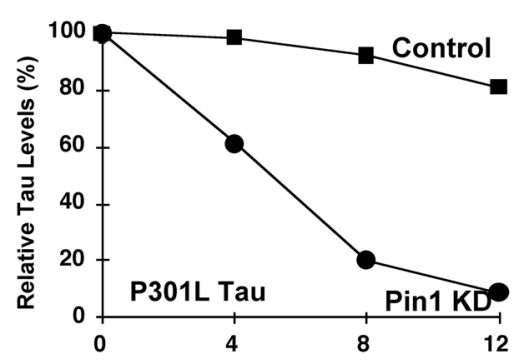

D

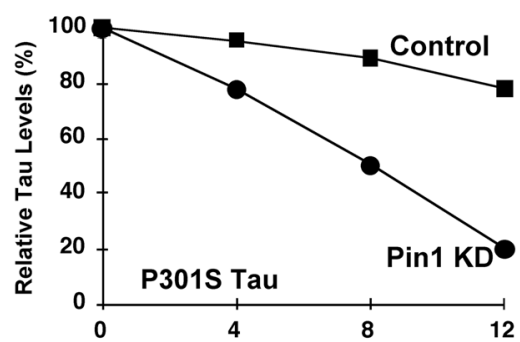

$\mathbf{F}$

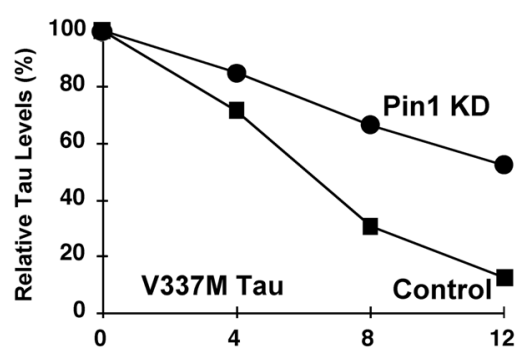

H

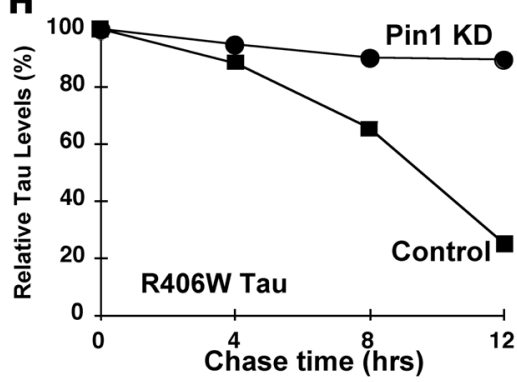

Figure 2

Effects of Pin 1 KD on protein stability of other selected FTDP-17 tau mutants. Pin1 KD or control SY5Y cells were transfected with indicated FTDP tau mutants followed by cycloheximide chase analysis for indicated times. Cell lysates were fractionated by SDS-PAGE and analyzed by immunoblotting with Tau5, anti-Pin1, or anti-actin antibodies (A, C, E, and $\mathbf{G})$. Tau levels were semiquantified from 3 different experiments using ImageQuant and normalized using actin as an internal control, with tau levels at 0 being defined as $100 \%$ (B, D, F, and $\mathbf{H})$.

cultures increases protein stability of WT tau are consistent with the previous findings that Pin1 KO leads to increased levels, aggregation, and filament formation of endogenous tau in the mouse brain (48). However, the findings that Pin1 KD decreases protein stability of P301L tau were rather unexpected. To further confirm these surprising findings and to examine their pathological significance, we examined the effects of Pin $1 \mathrm{KO}$ on tau-related phenotypes induced by P301L tau by analyzing littermates generated from crosses between P301L tau Tg mice and Pin1 KO mice. Total brain lysates and sarkosylinsoluble fractions were subjected to immunoblotting with $\mathrm{mAbs}$ that recognize all tau isoforms or specific tau phosphoepitopes and/or abnormal conformations present only in the neurofibrillary tangle (NFT) specific to AD (Figure 4, A and $\mathrm{B}$ ), a procedure widely employed to characterize tau Tg mice $(7-9,15,16,18-20,22,48)$.

Since Pin1 KO in mice induces tau hyperphosphorylation and other tau-related phenotypes only after 8-9 months (48), we analyzed the effects of Pin 1 KO on P301L tau at 6-8 months to avoid the complication of endogenous tau. As expected at this age, Pin $1 \mathrm{KO}$ did not have obvious effects on phosphorylation of endogenous tau, and P301L tau mice significantly overexpressed tau protein, as revealed by immunoblot with total tau mAb Tau5 (Figure 4A). Furthermore, tau protein was detected only in P301L tau mice when probed with human tau-specific $\mathrm{mAb}$ CP27 (Figure 4A). In total lysates, P301L tau overexpression resulted in aberrant tau hyperphosphorylation, as detected by pThr231-specific mAbs AT180, CP9, and CP17, and pSer202/ pThr205-specific mAb AT8 (Figure 4A). Furthermore, the mutant tau also existed in the NFTspecific conformations, as detected by the conformation-specific mAbs MC1 and AT100 and by aberrant mobility shift (Figure 4A). As shown previously $(7-9,15,16,18-20,22,48)$, although some endogenous tau isoforms were extracted from WT mouse brains by the sarkosyl extraction procedure, endogenous tau was not recognized by many phospho-specific mAbs in spite of a weak recognition by AT8 and AT100 (Figure 4B). However, there was considerable enrichment of hyperphosphorylated P301L tau with markedly retarded mobility and aberrant NFT conformations in the sarkosyl insoluble fraction of P301L $\mathrm{Tg}$ mice (Figure 4B). These results indicate that P301L Tg mice develop robust tau-related phenotypes, as reported $(7,15,22)$.

Pin1 KO greatly decreased overall levels of the P301L mutant (Figure 4A) and the aggregation of phosphorylated P301L tau into the sarkosyl insoluble fraction (Figure 4B) by approximately 4- to 5 -fold, consistent with the above findings that Pin $1 \mathrm{KD}$ or KO reduced the half-life of the mutant tau (Figures 1 and 3). Furthermore, at the age of 8 months, Pin $1 \mathrm{KO}$ in non-tau $\mathrm{Tg}$ mice did not induce aberrant endogenous tau phosphorylation, NFT conformations, and tau aggregation (Figure 4, A and B). However, Pin1 KO in P301L Tg mice dramatically reduced the accumulation of total hyperphosphorylated tau as well as tau in the abnormal NFT-specific conformations (Figure 4A). Moreover, Pin1 KO almost completely abolished the aggregation of phosphorylated tau into the sarkosyl insoluble fraction (Figure 4B). For example, Pin1 KO reduced AT180 and AT100 
A

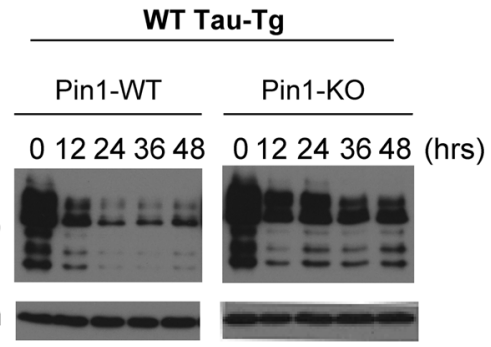

C

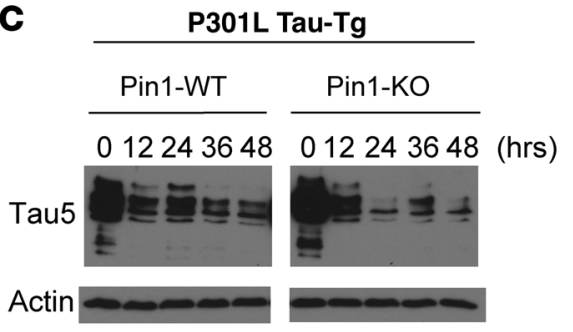

E

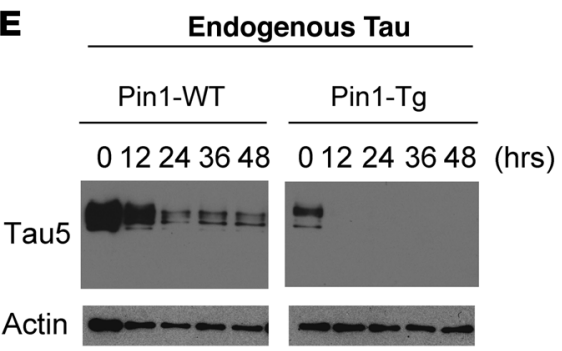

B

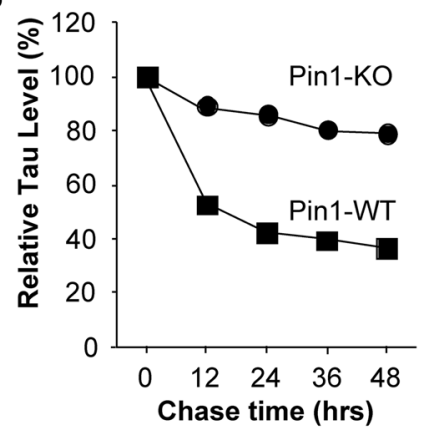

D

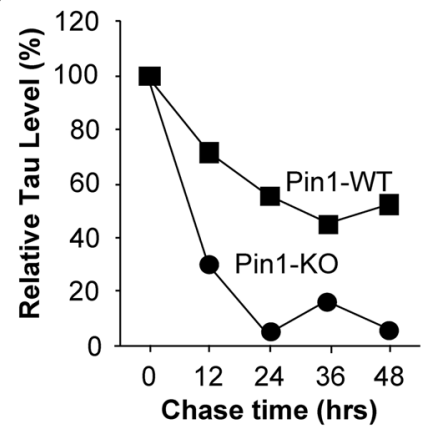

$\mathbf{F}$

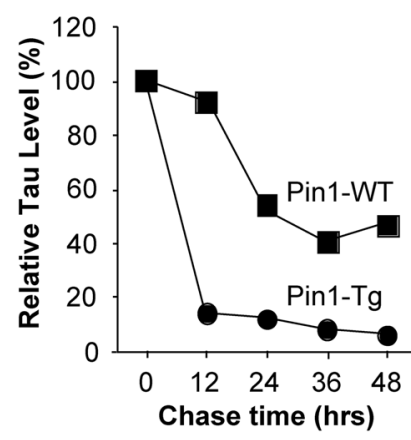

Figure 3

Pin1 has opposite effects on the protein stability of WT tau and P301L tau in brain-slice cultures. Brain tissue slices of $230 \mu \mathrm{m}$ were prepared and cultured from WT tau Tg mice in the presence (Pin1-WT) or absence (Pin1-KO) of Pin1 (A and B), from Tg P301L tau mice in the presence (Pin1-WT) or absence (Pin1-KO) of Pin1 (C and $\mathbf{D})$ ), or from Thy1Pin1 Tg mice (Pin1-Tg) or control non-Tg mice (Pin1-WT) (E and F). Cycloheximide was added to stop new protein synthesis and chased for indicated times. Brain slices were lysed in a buffer by sonication, followed by subjecting soluble protein lysates to immunoblotting with Tau5 or anti-actin antibodies (A, C, and E). Tau levels were semiquantified from 2 independent experiments using ImageQuant and normalized using actin as an internal control (B, D, and F).

immunoreactivities of tau in total lysates by 2 - to 3-fold and in the sarkosyl insoluble fraction by 5 - to 10 -fold. These results indicate that Pin $1 \mathrm{KO}$ dramatically reduces hyperphosphorylation, NFT conformations, and tau aggregation of P301L tau in mice.

To insure that the tau immunoreactivities observed on immunoblots are from neurons and also located in the expected subcellular compartments, we performed immunostaining analysis. Indeed, in contrast with non-Tg controls (data not shown), $\mathrm{Tg}$ P301L tau neurons exhibited strong immunoreactivities with AT180 in the soma region and axon of neurons in the cortex, brainstem, hippocampus, and spinal cord (Figure 4C and data not shown), as shown before $(7,9,15,19)$. Importantly, immunore-

activities with AT180 were dramatically reduced in P301L tau mice in the absence of Pin1 (Figure 4C). These results together indicate that Pin $1 \mathrm{KO}$ in mice almost completely suppresses the robust tauopathy phenotype induced by $\mathrm{P} 301 \mathrm{~L}$ tau.

Overexpression of Pin1 in postnatal neurons reduces protein stability of endogenous WT tau in mice. Together with the previous findings that Pin $1 \mathrm{KO}$ in mice is sufficient to cause the tauopathy phenotype (48), the above results indicate that Pin $1 \mathrm{KD}$ or $\mathrm{KO}$ has the opposite impact on tau protein stability and tauinduced phenotypes depending on whether the tau is WT or P301L in vitro and in vivo. Consequently, if Pin 1 is indeed a critical rate-limiting regulator in the development of the tauopathy phenotype induced by WT tau and its FTDP-17 mutant, we would expect that Pin 1 overexpression in the neuron would reduce the tauopathy phenotype in Tg WT tau mice but enhance the phenotypes in P301L Tg mice. To test this possibility, we generated $\mathrm{Tg}$ mice overexpressing Pin 1 in neurons. Given that long-term overexpression of Pin 1 in (proliferative) mammary glands disrupts the centrosome duplication checkpoint and induces breast cancer (44), we chose the Thy1.2 promoter to overexpress FLAG-tagged Pin1 in postnatal neurons (Figure 5, A-C) because it is active in neurons approximately 10 days after birth (59) so that the transgene does not interfere with the embryonic development of the brain.

To detect expression of FLAG-Pin1, we first performed immunoblotting analysis of various tissues, including brain, heart, liver, lung, pancreas, kidney, and testis. FLAG-Pin 1 with expected molecular weight was mainly expressed in brain tissues at levels only approximately 1 - to 2 -fold higher than endogenous levels (Figure 5D). There was faint FLAG-Pin 1 detected in testis, but none in many other tissues examined (Figure 5D). To determine whether Pin 1 is indeed overexpressed in neurons in the central nervous system, we performed immunohistochemical staining using both FLAG antibody and Pin 1 antibody on fixed tissue sections. Indeed, Pin 1 was detected mainly in neurons in the hippocampus, cortex, spinal cord, and other regions in both WT and Pin 1 Tg mice (Figure 5D and data not shown). In addition, the subcellular distribution of endogenous Pin 1 detected in WT animals was generally distributed to cell bodies and filamentous skeletal structures of neuronal processes in addition to the nucleus (Figure 5E), as shown (48). Exogenous FLAG-Pin 1 in Pin 1 Tg animals exhibited a similar distribution pattern compared with the endogenous protein in WT animals albeit at higher intensities (Figure 5F). Therefore, Pin 1 in these Tg mice is overexpressed only about 1-2 times over the endogenous levels and localized in the neuron as is endogenous Pin 1.

To determine whether Pin 1 overexpression affects endogenous tau in mouse brains, we examined Pin $1 \mathrm{Tg}$ mouse brains for any changes in tau phosphorylation and aggregation in the sarkosyl insoluble fraction and could not find any obvious differences between Pin1 Tg and non-Tg WT animals, even up to age 

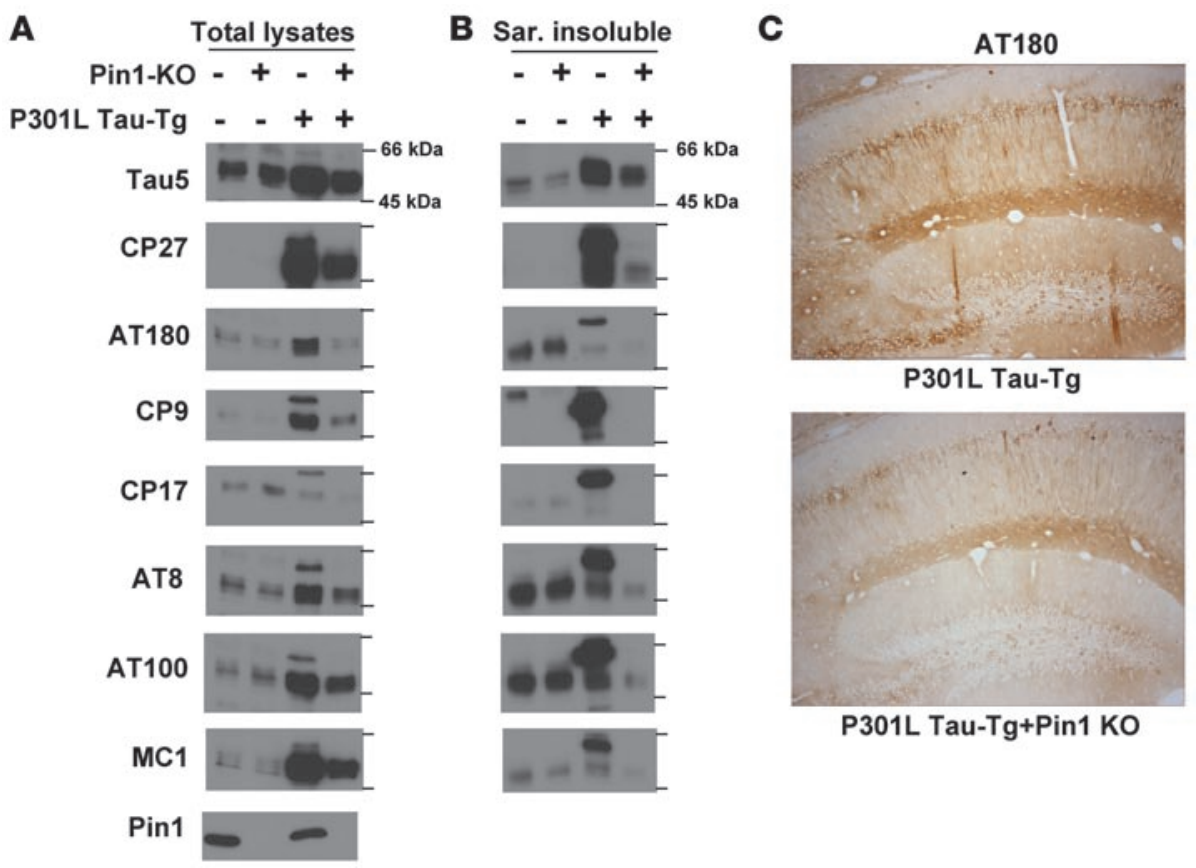

P301L Tau-Tg

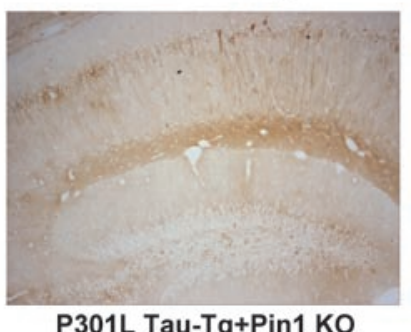

\section{Figure 4}

Pin1 KO in Tg P301L tau mice reduces tau hyperphosphorylation, NFT-specific conformations, and aggregation in the brain. (A and B) Brains of 6- to 8-month-old non- $\mathrm{Tg}$ or $\mathrm{Tg}$ P301L tau mice in the presence or absence of Pin1 KO, as indicated, were homogenized, followed by subjecting total lysates (A) or sarkosyl-insoluble (Sar. insoluble) fractions (B) to immunoblotting with different tau mAbs with the following specificities: Tau5, specific against total human and mouse tau; CP27, specific against total human tau; AT180, CP9, and CP17, specific against pThr231-tau; AT8, specific against pSer202/pThr205-tau; AT100, specific against pThr212-tau in the NFT-specific conformation; MC1, specific against tau in the NFT conformation. (C) Subcellular localization of phosphorylated tau. Mice were perfused and fixed, and the coronal hippocampal sections were subjected to immunohistochemical staining using AT180. Similar results were also obtained with AT100 (not shown). Original magnification, $\times 20$.

18 months, as assayed by immunoblotting and immunostaining using various antibodies (data not shown). However, we did observe that overall levels of endogenous tau were reduced in Pin 1 $\mathrm{Tg}$ mice, as compared with non-Tg controls (Figure 6A), suggesting that Pin 1 overexpression might reduce protein stability of endogenous mouse tau protein. To examine this possibility, we established brain-slice cultures from Thy1-Pin $1 \mathrm{Tg}$ mice and non$\mathrm{Tg}$ littermates followed by cycloheximide chase analysis to examine the half-life of endogenous tau in brain tissues. Compared with control mice, the half-life of tau was significantly reduced in Thy1Pin $1 \mathrm{Tg}$ brain tissues from approximately 24 hours to approximately 6 hours (Figure 3, E and F). These results indicate that $\mathrm{Tg}$ overexpression of Pin 1 in postnatal neurons at a moderate level is functionally active and sufficient to reduce endogenous tau protein stability. Thus, these results demonstrate that Pin 1 is a critical rate-limiting factor in controlling tau protein stability in vivo.

Neuronal Pin1 overexpression affects tau-related phenotypes in WT tau and $\mathrm{Tg}$ P301L tau mice in an opposite manner. To determine whether overexpression of Pin1 would affect the phenotypes of WT tau and P301L tau, we first crossed Pin 1 Tg mice (in FVB genetic background) and WT tau (18) or Tg P301L tau mice (7) (both in C57BL/6 background) to generate double-Tg mice. In addition, to reduce the influence of genetic background, we generated double-Tg mice on a pure C57BL/6 background. We used mice carrying only 1 copy of the forions by about 3- to 4-fold (Figure 6A). These inhibitory effects of Pin 1 overexpression on tau-related phenotypes are even more profound when analysis of sarkosyl insoluble fractions is considered (Figure $6 \mathrm{~B})$, with total tau5 immunoreactivity being reduced by 7 - to 8-fold and AT180 and AT100 immunoreactivities by 3- to 5-fold. Moreover, immunohistochemical staining with mAb AT180 further confirmed that Pin 1 overexpression reduced hyperphosphorylation of WT tau in neurons (Figure 6C and data not shown). These results together indicate that Pin 1 overexpression protects against the accumulation of total tau, tau hyperphosphorylation, NFT-specific conformations, and insoluble aggregation of WT tau.

In contrast, Pin 1 overexpression had the opposite effect on taurelated pathologies in P301L Tg mice (Figures 6 and 7). Young adult mice (6 months old) that were generated from Pin 1 and $\mathrm{Tg}$ P301L tau mouse crosses were subject to analysis for tau-related phenotypes. Compared with non-P301L mice, there was an obvious increase in tau levels in P301L mice, and interestingly, tau levels further increased by 2-to 3-fold in the presence of overexpressed Pin1 (Figure 7A), which is consistent with the findings that Pin1 $\mathrm{KD}$ or KO increases P301L tau stability (Figures 1 and 3). More interestingly, Pin 1 overexpression in Tg P301L tau mice significantly increased the accumulation of total hyperphosphorylated tau as well as tau in the abnormal NFT-specific conformations (Figure 7A). Moreover, these effects of Pin 1 on tau-related pheno- 
A

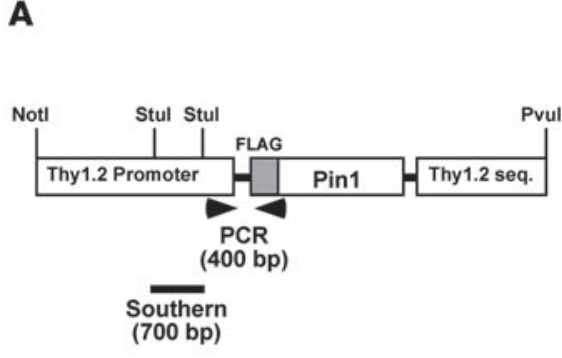

B
123456
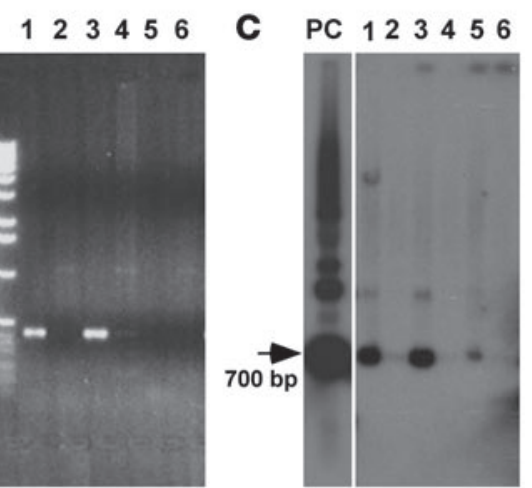

D $\quad$ WT Pin1-Tg

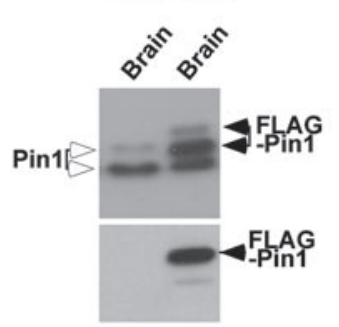

E
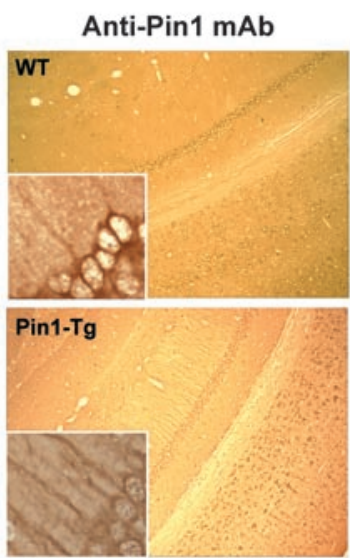

Pin1-Tg

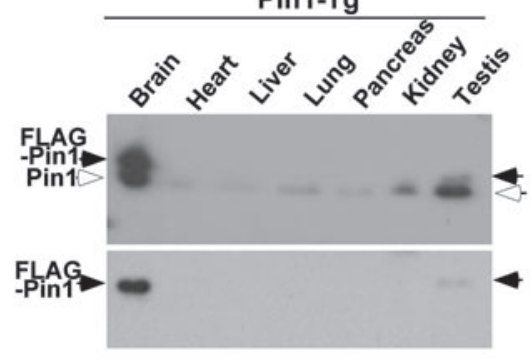

$\mathbf{F}$

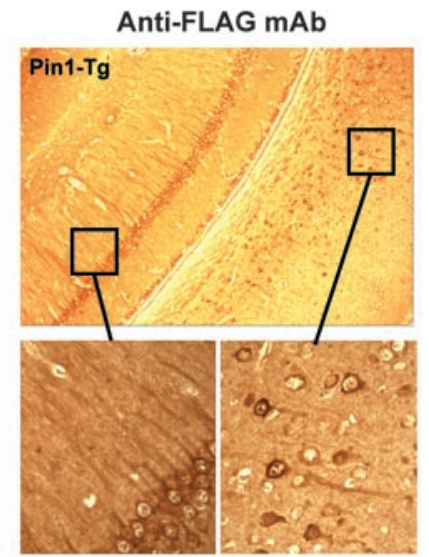

Figure 5

Generation of $\mathrm{Tg}$ mice that moderately overexpress Pin1 with subcellular localization similar to that of the endogenous protein. (A-C) Generation of Pin1 Tg mice. Pin1 cDNA with an N-terminal FLAG tag was subcloned into a murine Thy 1.2 genomic expression vector (A) followed by removing vector sequences of this construct before pronuclear microinjection into pure FVB mouse embryos. Founders were genotyped by PCR analysis (B) and genomic Southern analysis after Stul digestion (C) of DNA isolated from tail biopsies. (D) Moderate overexpression of FLAGPin1 selectively in the brain. Various Pin1 $\mathrm{Tg}$ mouse tissues and WT mouse brains were dissected and frozen in liquid nitrogen, followed by homogenization in SDS sample buffer before subjection to immunoblotting with antibodies against Pin1 and the FLAG epitope. (E and F) Similar subcellular localization of exogenous and endogenous Pin1 in the neuron. WT and Pin1 Tg mice were perfused and fixed, and the paraffin-embedded coronal sections were subjected to immunohistochemical staining with $\mathrm{mAb}$ against Pin1 (E) and the FLAG epitope (F). Original magnification, $\times 20$ (main photographs); ×40 (insets). types in $\mathrm{Tg}$ P301L tau mice were even more profound when the sarkosyl insoluble fractions were analyzed (Figure 7B). For example, Pin 1 overexpression increased AT180 and AT100 immunoreactivity in total lysates by 2 - to 3-fold and in the sarkosyl insoluble fractions by 3 - to 6 -fold. Finally, immunostaining confirmed that Pin 1 overexpression in Tg P301L tau mice dramatically increased accumulation of P301L tau hyperphosphorylation and NFT-specific conformations (Figure $7 \mathrm{C}$ and data not shown). These results indicate that Pin 1 overexpression greatly increases hyperphosphorylation, NFT conformations, and tau aggregation of P301L tau in mice, directly opposite of its effects in Tg WT tau mice.

Overexpression of Pin 1 affects spinal and peripheral neuropathies in WT tau and Tg P301L tau mice in an opposite manner. Overexpression of both WT tau and P301L tau in mice has been reported to trigger neuropathological changes, especially in spinal and peripheral neurons $(7,18)$. Aged Tg WT tau mice develop accumulation of silver-positive intraneuronal inclusions and vacuolar lesions in spinal cords accompanied by neurodegeneration (18). Tg P301L tau mice accumulate silver-positive axonal spheroids and suffer from ventral horn cell loss and axonal degeneration in spinal ventral roots, which correlates with their severe motor dysfunction (7). Given the opposite effects of Pin 1 overexpression on tau-related biochemical phenotypes in WT tau and Tg P301L tau mice, we asked whether Pin 1 overexpression has opposite effects on their spinal and peripheral neuropathies. We analyzed spinal cords and sciatic nerves isolated from age-matched WT, Tg WT tau, Pin1-Tg, and Pin $1 \mathrm{Tg} / \mathrm{tau} \mathrm{Tg}$ double-Tg mice, or Tg P301L tau and Pin $1 \mathrm{Tg} / \mathrm{Tg}$ P301L tau double-Tg mice. In 18-month-old Pin $1 \mathrm{Tg}$ mice, we did not detect any obvious neuronal lesions either by Bielschowsky silver staining of spinal cord (Figure 8, A and B) or by analysis of the sciatic nerves (Figure 9, A and B). In 18-month-old Tg WT tau mice, we observed neuropathological changes, as demonstrated by the presence of some silver-positive neurons in the ventral horn of spinal cord (Figure 8C) and several degenerating or demyelinated axons within the sciatic nerves, which were often engulfed by macrophages (Figure 9C). However, we observed neither silver-positive neurons in the spinal cord (Figure 8D) nor degenerated neurons within the sciatic nerve (Figure 9D) in Pin $1 \mathrm{Tg} / \mathrm{Tg}$ WT tau double$\mathrm{Tg}$ mice. The silver stain and neurodegeneration of Pin $1 \mathrm{Tg} / \mathrm{Tg}$ WT tau double-Tg mice were no different from those in age-matched 


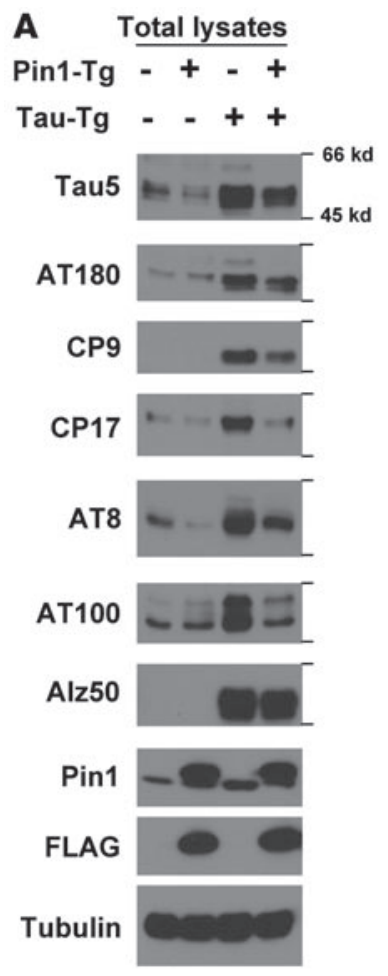

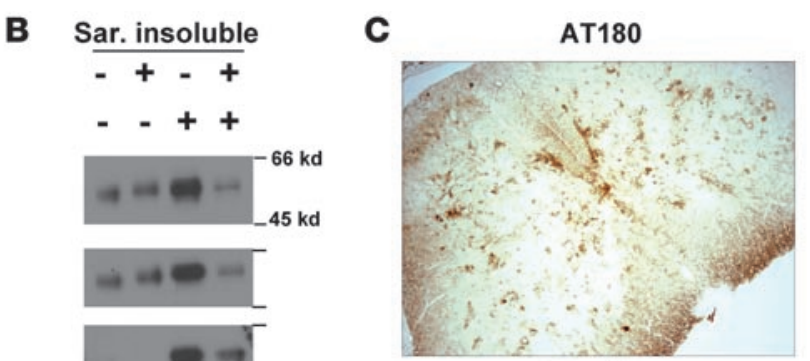

Tau-Tg
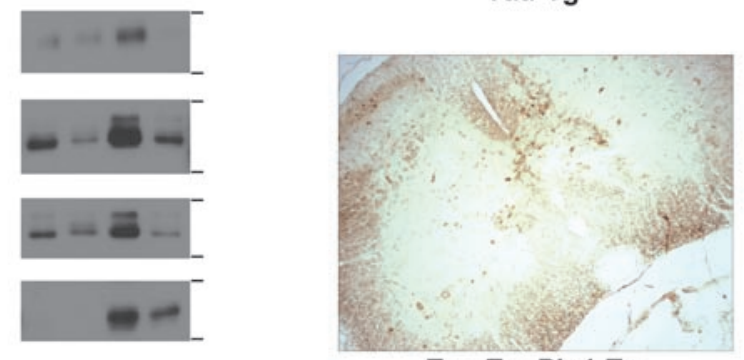

Tau-Tg+Pin1-Tg

\section{Figure 6}

Pin1 overexpression in WT tau mice reduces tau hyperphosphorylation, NFTspecific conformations, and aggregation in the brain. (A and $\mathbf{B}$ ) Brains of 1-yearold non-Tg or Tg WT tau mice in the presence or absence of Pin1 overexpression, as indicated, were homogenized, followed by subjecting total lysates (A) or sarkosylinsoluble fractions $(B)$ to immunoblotting with different tau mAbs with the following specificities: Tau5, specific against total human and mouse tau; AT180, CP9, and CP17, specific against pThr231-tau; AT8, specific against pSer202/pThr205-tau; AT100, specific against pThr212-tau in the NFT-specific conformation; Alz50, specific against tau in the NFT conformation. (C) Subcellular localization of phosphorylated tau. Mice were perfused and fixed; this was followed by subjection of coronal spinal cord sections to immunohistochemical staining with AT180. Original magnification, $\times 20$. non-tau Tg controls (Figures 8, A and B, and 9, A and B). These results indicate that $\mathrm{Pin} 1$ overexpression effectively inhibits spinal and peripheral neuropathies induced by WT tau.

Since we observed the onset of the motor dysfunction of the $\mathrm{Tg}$ P301L tau mice at around 12 months of age in the C57BL/6 background, we analyzed $\mathrm{Tg}$ P301L tau mice at 7 months of age and compared them with the age-matched Pin $1 \mathrm{Tg} / \mathrm{Tg}$ P301L tau double-Tg mice. At this early age, we detected relatively few silverpositive neurons in spinal cords of Tg P301L tau mice (Figure 8E) and few degenerating axons within their sciatic nerves (Figure 9E). However, we observed massive degeneration in age-matched Pin 1 $\mathrm{Tg} / \mathrm{Tg}$ P301L tau double-Tg mice, as demonstrated by Bielschowsky silver staining (Figure 8F) and by analysis of their sciatic nerves (Figure 9F). In addition, the peripheral neuropathy of the double$\mathrm{Tg}$ mice was accompanied by their severe motor dysfunction (data not shown). These results indicate that Pin 1 overexpression greatly enhances spinal and peripheral neuropathies induced by $\mathrm{P} 301 \mathrm{~L}$ tau. Furthermore, the opposite effects of Pin 1 overexpression on hyperphosphorylation, NFT conformations, and tau aggregation of WT tau and P301L tau in Tg mice correlate well with the appearance of neurodegenerative phenotypes in spinal cords of the mice.

\section{Discussion}

We have shown that Pin $1 \mathrm{KD}$ or $\mathrm{KO}$ increases protein stability of WT tau in vitro and in vivo and that neuronal Pin 1 overexpression greatly inhibits the tauopathy phenotype in Tg WT tau mice. These findings are consistent with the previous findings that Pin 1 $\mathrm{KO}$ alone is sufficient to induce the tauopathy phenotype in mice. However, unexpectedly, we found that Pin $1 \mathrm{KD}$ or KO reduces protein stability of P301L tau in vitro and in vivo. This finding has been further substantiated by our demonstration that Pin $1 \mathrm{KO}$ almost completely abrogates the robust tau-related phenotypes in $\mathrm{Tg}$ P301L tau mice. In contrast, neuronal Pin1 overexpression drasti- cally exacerbates tau-related phenotypes and neurodegeneration in $\mathrm{Tg}$ P301L tau mice. These results have demonstrated for what we believe is the first time that Pin 1 has diametrically opposite impacts on the tauopathy phenotype depending on whether the tau is WT or P301L mutant. Given that most human tauopathies such as AD do not have tau mutations, these results indicate that it is critical to use relevant tau models for investigating the development and treatment of different tauopathies. Furthermore, our results suggest that Pin1 upregulation might be beneficial for $\mathrm{AD}$, whereas its inhibition might be helpful for patients carrying the P301L tau mutation.

It has been shown that Pin 1 is greatly induced during neuronal differentiation and is highly expressed in most neurons in the brain but is especially low in the neurons that are most vulnerable to neurodegeneration in $\operatorname{AD}(48,49)$. Furthermore, Pin 1 has been shown to be inactivated or downregulated by oxidative modifications or genetic polymorphisms in human $\mathrm{AD}(48,50-52)$. Importantly, Pin 1 binds to and isomerizes phosphorylated tau to restore tau biological function and to promote tau dephosphorylation $(46,47)$. Moreover, Pin $1 \mathrm{KO}$ in mice induces the tauopathy phenotype, $A \beta$-related phenotypes, and neurodegeneration in an agedependent manner, as seen in human $\operatorname{AD}(39,48)$. These results indicate that loss of Pin 1 function contributes to the tauopathy phenotype in $\mathrm{AD}$, but whether increasing Pin1 function can suppress tau-related phenotypes is unknown.

To address this question, we generated $\mathrm{Tg}$ mice that overexpress Pin 1 in postnatal neurons using the mouse Thy 1.2 promoter. Exogenous Pin 1 was expressed specifically in the neurons of the central nervous system at approximately 1- to 2-fold higher than endogenous levels and was localized to the same subcellular compartments as endogenous protein (Figure 5). This moderate level of Pin 1 overexpression alone did not have any detectable deteriorative effects but was sufficient to reduce protein stability of endogenous mouse tau (Figure 3, E and F). More impressively, when 


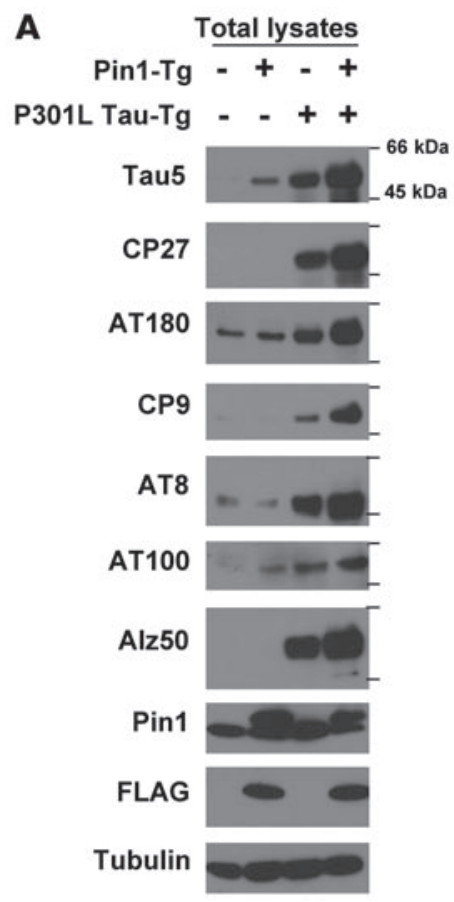

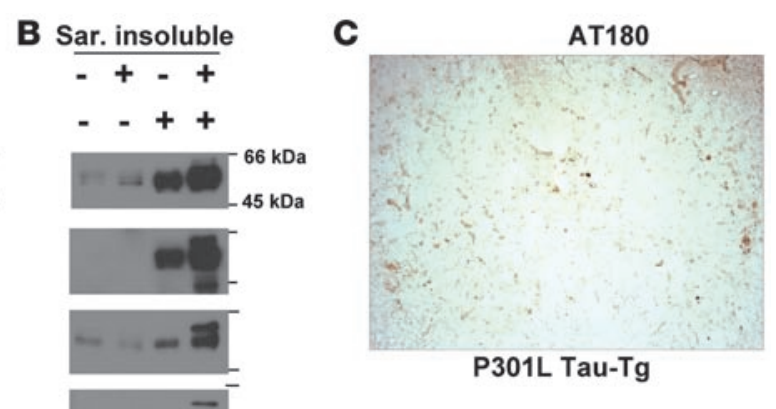

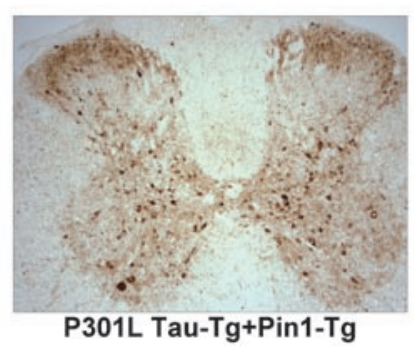

\section{Figure 7}

Pin1 overexpression in P301L tau mice increases tau hyperphosphorylation, NFT-specific conformations, and aggregation in the brain. (A and B) Brains of 6-month-old non-Tg or Tg P301L tau mice in the presence or absence of Pin1 overexpression, as indicated, were homogenized, followed by subjection of total lysates $(\mathbf{A})$ or sarkosyl-insoluble fractions (B) to immunoblotting with different tau mAbs with the following specificities: Tau1 and Tau5, specific against total human and mouse tau; CP27, specific against total human tau; AT180 and CP9, specific against pThr231-tau; AT8, specific against pSer202/pThr205tau; AT100, specific against pThr212tau in the NFT-specific conformations; Alz50, specific against tau in the NFT conformations. (C) Subcellular localization of phosphorylated tau. Mice were perfused and fixed; this was followed by subjecting coronal spinal cord sections to immunohistochemical staining with AT180. Original magnification, $\times 20$. crossed to mice overexpressing human WT tau, Pin 1 overexpression strongly suppressed tau-related phenotypes, including tau hyperphosphorylation, NFT conformations, and tau aggregation as well as neurodegeneration (Figures 6, 8, and 9). These results from Pin $1 \mathrm{KO}$ and Pin $1 \mathrm{Tg}$ mice have conclusively demonstrated that Pin 1 is a critical rate-limiting factor in controlling WT tau protein stability and its tauopathy phenotype, which is found in $\mathrm{AD}$ and most other tauopathies, and also provide what we believe is the first in vivo evidence that upregulating Pin 1 function is useful for inhibiting the tauopathy phenotype in AD.

The surprising findings of our current study are that the effects of Pin 1 on P301L tau are diametrically opposite to those on WT tau in vitro and in vivo. Even though tau mutations have not been identified in $\mathrm{AD}, \mathrm{Tg}$ P301L tau mice have been tacitly assumed to be a preferred $\mathrm{AD}$ model due to their robust tauopathy phenotype (7-14). Since the P301L mutation does not affect tau binding to Pin1 (46), we assumed that Pin1 could regulate P301L tau in the same way as WT tau. Indeed, Pin 1 KD significantly affects protein stability of P301L tau, with the effects depending on the presence of the Pin1-binding site in tau, similarly to WT tau (Figure 1). However, unlike WT tau, Pin $1 \mathrm{KD}$ or KO did not increase but rather decreased P301L tau stability (Figures 1 and 3). As for other FTDP-17 tau mutants, Pin1 KD also decreased protein stability of P301S tau but increased protein stability of V337M tau and R406W tau (Figure 2), indicating that the effects of Pin 1 on FTDP-17 tau mutants likely depend on the identity of the tau mutations, as expected. To confirm these findings, we focused on P301L by ablating the Pin 1 gene or overexpressed Pin 1 in postnatal neurons in P301L tau mice and examined their effects on tau-related phenotypes, given that P301L tau mouse models are well established and widely used. Indeed, Pin1 KO in Tg P301L tau mice reduced protein stability and almost completely abolished hyperphosphorylation, NFT-specific conformations, and aggregation of the mutant tau (Figure 4), whereas Pin 1 overexpression had the opposite effects and also greatly enhanced neu- rodegeneration in P301L tau mice (Figures 7-9). These opposite effects of Pin 1 overexpression in WT tau mice and P301L tau mice are unlikely due to 1 extra microtubule-binding repeat present in P301L tau mice because Pin $1 \mathrm{KD}$ also had the same opposite effects on both WT tau and P301L tau containing 4 microtubule-binding repeats (Figure 1). These in vitro and in vivo results together indicate that Pin 1 has the diametrically opposite impact on the tauopathy phenotype depending on whether the tau is WT or P301L mutant (Figure 10). Given that most human tauopathies such as $\mathrm{AD}$ do not have tau mutations, our results indicate that it is critical to use Tg WT tau mice or to develop more relevant models for investigating the development and treatment of the tauopathy phenotype in $\mathrm{AD}$. Moreover, our results suggest that inhibiting Pin1 function might be beneficial for FTDP-17 patients carrying P301L tau mutation.

Pin 1 has been shown to affect a spectrum of protein activities, often in the opposite manner depending on substrates, such as decreasing or increasing protein stability, which might depend on whether specific pSer/Thr-Pro motifs are in trans or cis (42). Indeed, Pin1-catalyzed conformational changes between the 2 distinct cis and trans isomers have been visualized by NMR for APP and also shown to promote nonamyloidogenic APP processing and to reduce $A \beta$ levels (39). In the case of tau, Pin1-catalyzed conformational changes have been shown to restore tau microtubule function and to promote tau dephosphorylation. Our results have shown that Pin 1 also promotes tau degradation in WT protein, probably via inducing conformational changes (Figure 10A). This is consistent with a common theme that Pin 1 often modulates its substrates via multiple mechanisms or acts on multiple targets to help drive certain cellular processes in one direction (42). However, in the case of P301L tau, Pin 1 inhibits tau degradation (Figure 10B). Given the well-documented role of tau protein levels in the development of tauopathies, these differential effects of Pin 1 on WT tau and P301L tau would be eventually manifested in their ability to aggregate and form NFTs and to induce neurodegeneration (Figure 10). 


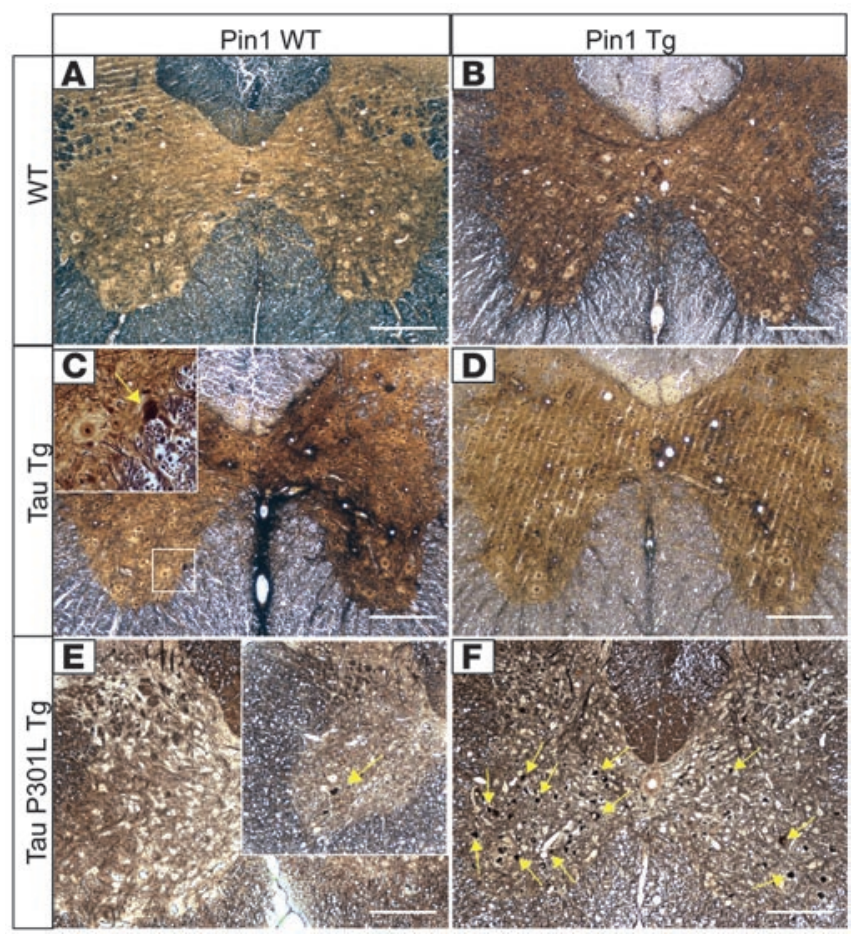

We do not yet know how the P301L mutation alters tau regulation so dramatically that the regulation of P301L tau by Pin 1 becomes opposite to that of WT tau due to the lack of available tau structures. However, this is likely related to the fact that the P301L mutation would have a significant impact on the tau structure and that certain pSer/Thr-Pro motifs can exist in 2 completely distinct cis and trans conformations due to a 5-carbonyl ring of Pro in the peptide bond $(42,60)$. Tau phosphorylation has been shown to promote tau degradation via chaperone-interacting protein/Hsp70 (31-33) or inhibit tau degradation (34). Interestingly, F-box proteins, which target phosphorylated proteins to ubiquitin-mediated proteolysis, bind to pSer/Thr-Pro motifs only in trans, but not cis (61). Given the dramatic and opposite effects of manipulating Pin1 function on protein stability of WT tau and P301L tau and their ability to induce the tau-related phenotypes in cell cultures and animals, we hypothesize that proteins targeting phosphorylated tau for degradation might bind to certain pSer/Thr-Pro motif(s) in tau only in 1 conformation and Pin1-catalyzed cis/trans isomerization would promote this interaction to promote degradation of WT tau (Figure 10, A and B). However, in the case of P301L tau, the mutation might somehow render the pSer/Thr-Pro motifs in tau to be favored in the other conformation, and Pin1-catalyzed

\section{Figure 9}

Opposite effects of Pin1 overexpression on peripheral neuropathies of WT tau and Tg P301L tau mice. Analysis of semithin sections of the sciatic nerves revealed presence of degenerating axons (arrows) often engulfed by macrophages (C, inset) in 18-month-old Tg WT tau mice (C) but not in the age-matched Pin1/tau double-Tg mice (D), non- Tg WT (A), or Pin1 Tg mice (B). On the contrary, few neuronal lesions were detected in sciatic nerves (E) of 7-month-old Tg P301L tau mice, while overexpression of Pin1 and P301L tau caused massive neurodegeneration in the sciatic nerves of the age-matched double- $\mathrm{Tg}$ mice. (F). Arrowheads point to demyelinated axon, and arrows point to macrophages clearing myelin debris. Scale bars: $20 \mu \mathrm{m}$.

\section{Figure 8}

Opposite effects of Pin1 overexpression on spinal neuropathies of WT tau and Tg P301L tau mice. Bielschowsky silver staining revealed silver-positive neurons in spinal cords of 18-month-old Tg WT tau mice (C, inset) but not in the Pin1/tau double-Tg (D), WT (A), or Pin1 Tg mice (B). On the contrary, few neuronal lesions were detected in spinal cords (E) of 7-month-old Tg P301L tau mice, while overexpression of Pin1 and the P301L tau caused massive silver-positive neurodegeneration in the spinal cord in the age-matched double-Tg mice (F). Arrows point to silver-positive degenerated neurons. Scale bars: $100 \mu \mathrm{m}$.

trans/cis isomerization would prevent phosphorylated P301L tau from being targeted for degradation (Figure 10, C and D).

In this model, although both WT tau and P301L tau are probably phosphorylated on certain Ser/Thr-Pro motifs such as the Thr231Pro motif in trans due to the conformation specificity of kinases (42), the pThr231-Pro motif in WT tau might have a tendency to be in the cis conformation due to local structural constraints after phosphorylation, as shown for APP (62). However, Pin1 would rapidly reestablish equilibrium if the trans (or cis) population were suddenly depleted by certain degradation pathways due to the extremely slow uncatalyzed isomerization rate. Therefore, in the nonequilibrium cellular environment, Pin 1 overexpression might greatly accelerate the cis to trans isomerization to promote tau degradation (Figure 10A), whereas Pin 1 inhibition might allow a higher concentration of cis pThr231-Pro motif to be present for a longer time, which might inhibit tau degradation (Figure 10B). However, in the case of P301L tau, the mutation might somehow render certain pSer/Thr-Pro motifs in tau such as the pThr231Pro motif to have a tendency to be in the trans conformation after phosphorylation. Therefore, Pin 1 inhibition might allow a higher concentration of trans pThr231-Pro motif to be present for a lon-

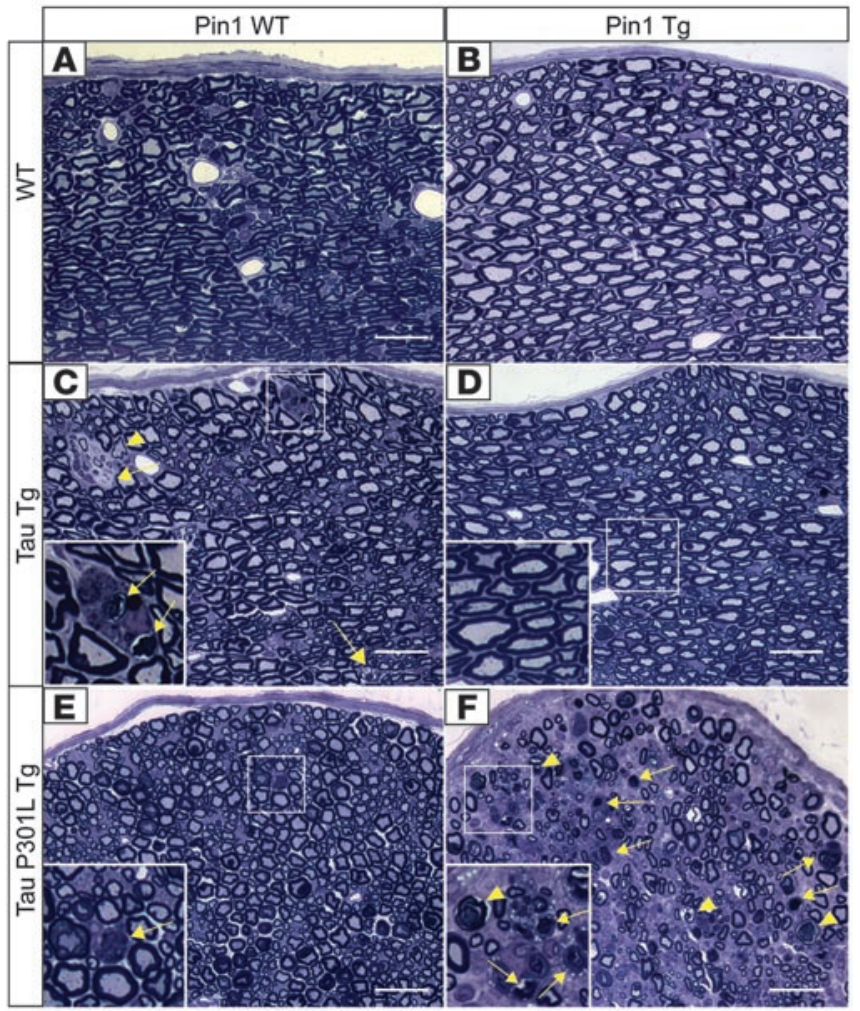


A

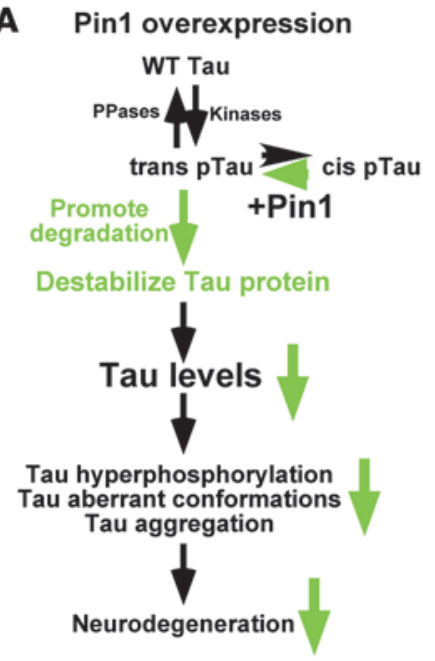

C

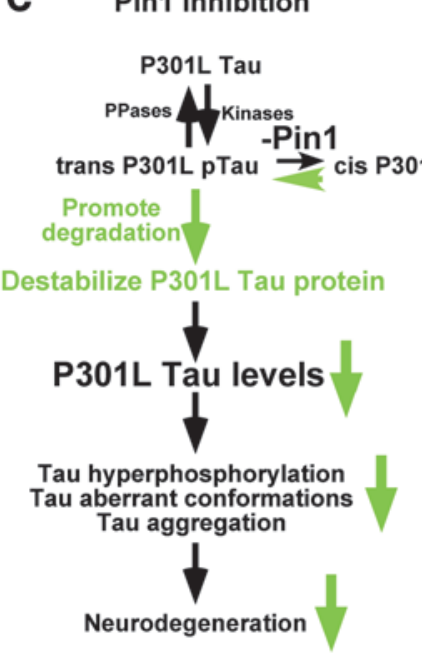

B Pin1 Inhibition

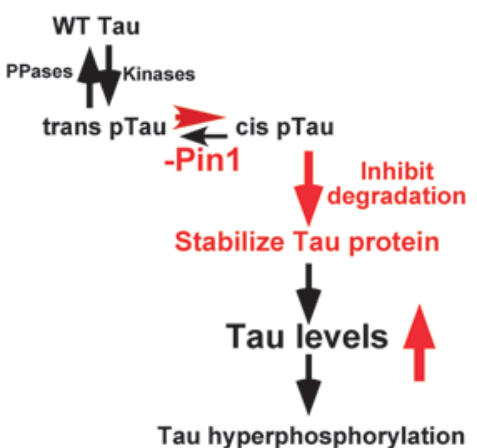

Tau hyperphosphorylation
Tau aberrant conformations Tau aggregation

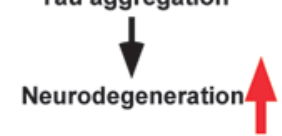

D Pin1 overexpression

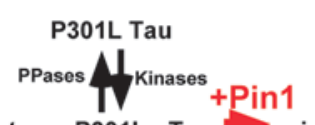

trans P301L pTau 2 cis P301L pTau

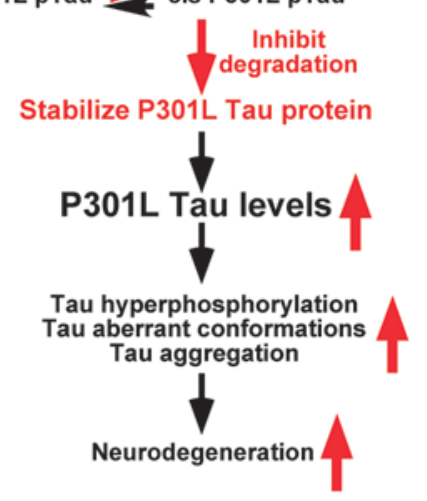

\section{Figure 10}

A model for the diametrically opposite impact of Pin1 on tau levels and tauopathy phenotype depending on whether the tau is WT or P301L mutant. Our results have shown that Pin1 has the opposite impact on the ability to induce the tauopathy phenotype. Although both WT tau and P301L tau are likely phosphorylated on certain Ser/Thr-Pro motifs, such as the Thr231-Pro motif in trans by upstream kinases, the pThr231-Pro motif in WT tau might have a tendency to be in the cis conformation due to local structural constraints. Pin1 overexpression might greatly accelerate the cis to trans isomerization to promote tau degradation (A). When Pin1 is inhibited, the cis pThr231-Pro motif might not be isomerized to trans in a timely manner, which might inhibit tau degradation (B). However, in the case of P301L tau, the mutation might somehow change tau conformation so that the pThr231-Pro motif might have a tendency to be in trans, which might promote $\mathrm{P} 301 \mathrm{~L}$ tau degradation when Pin1 is inhibited (C). However, Pin1 overexpression might accelerate the trans to cis isomerization to inhibit P301L tau degradation (D). PPases, protein phosphatases. ger time, which might promote P301L tau degradation (Figure 10C), whereas Pin 1 overexpression might accelerate the trans to cis isomerization to inhibit P301L tau degradation (Figure 10D).

This model is consistent with our findings that overexpression of Pin 1 reduces WT tau stability and abolishes its ability to induce the tauopathy phenotype but increases P301L tau stability and enhances its ability to induce the tauopathy phenotype. In contrast, Pin $1 \mathrm{KO}$ or KD has the opposite effects both on WT tau and P301L tau. Furthermore, the findings that Pin 1 KD increased protein stability of WT, V337M, or R406W tau but decreased protein stability of P301L or P301S tau are coincidently consistent with the fact that both P301L and P301S but not V337M tau and R406W tau mutations would allow P301 to exist only in the trans, but not cis, conformation, although further experiments are needed to determine the impact of the P301 mutations on tau structure, especially related to its Pin1-targeting site. Moreover, this is also consistent with the previous and current findings that manipulating Pin 1 specifically affects the accumulation of an AD pathological conformation of the pThr231-Pro-containing tau in brain that is recognized by $\mathrm{TG} 3$, a $\mathrm{mAb}$ that probably recognizes only the cis pThr231-Pro motif $(48,63)$. Therefore, cis and trans conformations of the pThr-Pro motif in tau and APP are distinct not only in their structures but also in their biological function or pathological consequences. Therefore, the events subsequent to tau phosphorylation such as degradation and dephosphorylation may be dynamically regulated, which are required under physiological conditions for cytoskeletal reorganization or vesicle transport along microtubule. The distinct properties of WT tau and P301L mutant tau may be magnified when Pin 1 is added into the equation because of its pivotal role in catalyzing tau between 2 distinct conformations after phosphorylation (46-48). Further studies on Pin1-catalyzed conformational regulation of tau and its mutants and its biological and pathological significance should help elucidate the molecular events leading to tauopathies and might also lead to the development of new therapies.

In summary, we have shown that the prolyl isomerase Pin 1 acts on both WT tau and P301L tau but has completely opposite results. Pin 1 KD or KO greatly increases WT tau protein stability and its ability to induce the tauopathy phenotype but drastically decreases P301L tau protein stability and its ability to induce the tauopathy phenotype in mice, whereas Pin1 overexpression has the opposite effect. These results indicate that although WT tau deregulation, as seen in $\mathrm{AD}$ and most tauopathies, and P301L tau mutation, as seen in some FTDP-17 patients, cause similar tau-related pathologies, 
the regulatory mechanisms that modulate the development of these tau-related phenotypes are quite different, pointing to the importance of using relevant models for investigating the development and treatment of human tauopathies. Moreover, our results suggest that Pin1 upregulation might be beneficial for $\mathrm{AD}$, whereas its inhibition might be helpful for patients with P301L tau.

\section{Methods}

Generation of Pin $1 \mathrm{KD}$ cells and expression constructs. HT1080 cells were infected with Pin 1 siRNA or control siRNA retroviruses, as described (53). For generating stable Pin1 KD SH-SY5Y cells, Pin1 shRNA lentiviral vector (Open Biosystems) was transfected together with VSV-G- and gag-pol-expressing plasmids (Open Biosystems) into 293T cells and stable clones selected using $0.5-1 \mu \mathrm{g} / \mathrm{ml}$ puromycin (53). To generate lentiviruses expressing WT tau and P301L tau, WT tau and P301L tau were cloned into the self-inactivating internal ribosome entry site-GFP lentiviral vector through SpeI, SalI restriction sites, as described (64).

Analysis of tau protein stability in cell cultures. Pin $1 \mathrm{KD}$ or control cells were transfected with WT tau or P301L tau constructs for 12 hours, and cycloheximide $(100 \mu \mathrm{g} / \mathrm{ml})$ was added. Cells were harvested at each time point, and total lysates were analyzed by immunoblotting with $\mathrm{mAb}$ against total tau or actin. For assaying tau stability in primary neurons, cortical neurons were isolated from E17.5 WT and Pin1 $1^{-/-}$embryos and cultured in Neurobasal Medium (Invitrogen) (65). After 2 days in culture, primary neurons were infected with lentiviruses expressing either WT tau or P301L tau. Tau stability assay was performed 3 days after infection after addition of $100 \mu \mathrm{g} / \mathrm{ml}$ cycloheximide, followed by assaying tau stability by immunoblotting. The immunoblots were scanned and semiquantitated using the software NIH Image 1.6.2, as described (66).

Generation of Pin $1 \mathrm{Tg}$ mice and mouse crosses. To generate Pin $1 \mathrm{Tg}$ mice, Pin1 cDNA with an N-terminal FLAG tag was subcloned into a murine Thy 1.2 genomic expression vector pR5 (provided by Li-Huei Tsai, The Picower Institute, Massachusetts Institute of Technology, Boston, Massachusetts, USA) (67), followed by removal of vector sequences of this construct before microinjection. $\mathrm{Tg}$ mice were produced by pronuclear injection of pure FVB mouse embryos. Founders were identified by PCR analysis of lysates from tail biopsies and then intercrossed with pure FVB mice to establish lines. Expression of FLAG-Pin 1 was confirmed by immunoblotting and immunostaining analyses. To generate Pin $1 \mathrm{Tg}$ mice in pure C57BL/6 background, Pin $1 \mathrm{Tg}$ mice in FVB background were backcrossed with C57BL/ 6 mice for 6 generations. $\mathrm{Tg}$ tau $3 \mathrm{R}$ mice in C57BL/6 background (18) and Tg P301L tau 4R mice in C57BL/ 6 background (7) (purchased from Taconic) were as described, respectively. Animal care and use for these experiments was approved by the Standing Committee on Animals at Harvard Medical School.

To generate P301L tau mice in the presence or absence of Pin1, Pin $1^{+/-}$ mice heterozygous on a mixed $129 / \mathrm{SvJ}$ and C57BL/6 background (68) were first crossed with P301L tau mice, which were subsequently crossed with Pin $1^{+/-}$heterozygous mice to obtain P301L tau Tg in Pin1 WT or KO mice with the expected controls. Since Pin $1^{+/-}$heterozygous mice are indistinguishable from WT mice $(68,69)$, Pin 1 WT or homozygous KO mice were used. All the Tg mice analyzed carried only a single copy of the respective transgenes, and littermates from the same crosses were used, with the exception of some experiments involving Pin1 KO mice, in which different litters at the same ages were pooled due to difficulties in breeding Pin1 KO mice. Similar phenotypes were observed in at least 3 different mice of the same genotypes in multiple experiments.

Organotypic brain-slice cultures. Organotypic brain-slice cultures were prepared essentially as described (70). In brief, brains from adult mice were removed and cut into coronal slices of $230-\mathrm{mm}$ thickness with a vibratome. Slices containing the cerebral cortex and hippocampus were chosen. The slices were placed onto Millicell-CM culture inserts (Millipore) in 6-well culture plates with culture medium.

Immunoblotting analysis of tau in the mouse brain. Total lysates and sarkosylinsoluble extracts were obtained as described $(7,15,18,48)$. Alz50, MC1, $\mathrm{CP} 9, \mathrm{CP} 17$, and CP27 mAbs against tau were gifts from P. Davies (Albert Einstein College of Medicine, Yeshiva University, New York, New York, USA). AT8, AT100, and AT180 (Endogen; Pierce Biotechnology), Tau1 (Chemicon), and Tau5 (BioSource) antibodies were purchased.

Immunohistochemical and histological analysis. Immunohistochemical analyses of mice were as described $(7,15,18,48)$. Bielschowsky silver staining was performed on $10-\mu \mathrm{m}$ transversal sections of paraffin-embedded lumbar spinal cord as described previously (71). For analysis of the sciatic nerves, the dissected nerves were embedded in EPON, and 1- $\mu \mathrm{m}$ semithin sections were stained with toluidine blue.

Statistics. Values are presented as mean \pm SD, and differences between groups were analyzed by 2 -sided Student's $t$ test. A $P$ value of less than 0.05 was considered statistically significant.

\section{Acknowledgments}

We thank L. Cantley, B. Neel, and L. Harrington for their intellectual input and constructive suggestions; R. Bronson for processing mouse histology and helping analyze neurodegenerative pathology; P. Davies for providing some tau antibodies; L.-H. Tsai, X. He, and M. Karin for providing DNA constructs; and C. Soohoo for editing the manuscript. J. Lim is a Human Frontiers Science Program fellow. The work was supported by NIH grants AG22082 and AG17870 and a philanthropic gift from Epix Pharmaceuticals to K.P. Lu.

Received for publication October 23, 2007, and accepted in revised form February 29, 2008.

Address correspondence to: Kun Ping Lu, Cancer Biology Program, Department of Medicine, Beth Israel Deaconess Medical Center, Harvard Medical School, 77 Avenue Louis Pasteur, NRB 1030, Boston, Massachusetts 02115, USA. Phone (617) 667-4143; Fax (617) 667-0610; E-mail: klu@caregroup.harvard.edu.

Jormay Lim's present address is: Institute of Molecular and Cell Biology, Proteos, Singapore.

Yih-Cherng Liou's present address is: Department of Biochemistry, National University of Singapore, Singapore.

Anyang Sun's present address is: Institute of Neurobiology, Fudan University, Shanghai, People's Republic of China.

Martin Balastik, Tae Ho Lee, Kazuhiro Nakamura, Yih-Cherng Liou, and Anyang Sun contributed equally to this work.
24:1121-1159

4. Stoothoff, W.H., and Johnson, G.V. 2005. Tau phosphorylation: physiological and pathological consequences. Biochim. Biophys. Acta. 1739:280-297.

5. Mawal-Dewan, M., et al. 1994. The phosphorylation state of tau in the developing rat brain is regulated by phosphoprotein phosphatases. J. Biol.
Chem. 269:30981-30987.

6. Matsuo, E.S., et al. 1994. Biopsy-derived adult human brain tau is phosphorylated at many of the same sites as Alzheimer's disease paired helical filament tau. Neuron. 13:989-1002.

7. Lewis, J., et al. 2000. Neurofibrillary tangles, amyotrophy and progressive motor disturbance in mice 
expressing mutant (P301L) tau protein. Nat. Genet. 25:402-405.

8. Lewis, J., et al. 2001. Enhanced neurofibrillary degeneration in transgenic mice expressing mutant tau and APP. Science. 293:1487-1491.

9. Gotz, J., Chen, F., van Dorpe, J., and Nitsch, R.M. 2001. Formation of neurofibrillary tangles in P3011 tau transgenic mice induced by Abeta 42 fibrils. Science. 293:1491-1495.

10. Oddo, S., et al. 2003. Triple-transgenic model of Alzheimer's disease with plaques and tangles: intracellular Abeta and synaptic dysfunction. Neuron. 39:409-421.

11. Noble, W., et al. 2003. Cdk5 is a key factor in tau aggregation and tangle formation in vivo. Neuron. 38:555-565

12. Lin, W.L., Lewis, J., Yen, S.H., Hutton, M., and Dickson, D.W. 2003. Filamentous tau in oligodendrocytes and astrocytes of transgenic mice expressing the human tau isoform with the $\mathrm{P} 301 \mathrm{~L}$ mutation. Am. J. Pathol. 162:213-218.

13. Santacruz, K., et al. 2005. Tau suppression in a neurodegenerative mouse model improves memory function. Science. 309:476-481.

14. Ramsden, M., et al. 2005. Age-dependent neurofibrillary tangle formation, neuron loss, and memory impairment in a mouse model of human tauopathy (P301L). J. Neurosci. 25:10637-10647.

15. Allen, B., et al. 2002. Abundant tau filaments and nonapoptotic neurodegeneration in transgenic mice expressing human P301S tau protein. J. Neurosci. 22:9340-9351.

16. Gotz, J., et al. 1995. Somatodendritic localization and hyperphosphorylation of tau protein in transgenic mice expressing the longest human brain tau isoform. EMBO J. 14:1304-1313.

17. Goedert, M., and Hasegawa, M. 1999. The tauopathies: toward an experimental animal model. Am.J. Pathol. 154:1-6.

18. Ishihara, T., et al. 1999. Age-dependent emergence and progression of a tauopathy in transgenic mice overexpressing the shortest human tau isoform. Neuron. 24:751-762.

19. Tanemura, K., et al. 2002. Neurodegeneration with tau accumulation in a transgenic mouse expressing V337M human tau. J. Neurosci. 22:133-141.

20. Spittaels, K., et al. 1999. Prominent axonopathy in the brain and spinal cord of transgenic mice overexpressing four-repeat human tau protein. Am.J. Pathol. 155:2153-2165.

21. Gotz, J. 2001. Tau and transgenic animal models. Brain Res. Brain Res. Rev. 35:266-286.

22. Gotz, J., Chen, F., Barmettler, R., and Nitsch, R.M. 2001. Tau filament formation in transgenic mice expressing P301L tau. J. Biol. Chem. 276:529-534.

23. Illenberger, S., et al. 1998. The endogenous and cell cycle-dependent phosphorylation of tau protein in living cells: implications for Alzheimer's disease. Mol. Biol. Cell. 9:1495-1512.

24. Pelech, S.L. 1995. Networking with proline-directed protein kinases implicated in tau phosphorylation. Neurobiol. Aging. 16:247-256; discussion 257-261.

25. Goedert, M., et al. 2000. Reduced binding of protein phosphatase $2 \mathrm{~A}$ to tau protein with frontotemporal dementia and parkinsonism linked to chromosome 17 mutations. J. Neurochem. 75:2155-2162.

26. Sontag, E., et al. 1996. Regulation of the phosphorylation state and microtubule-binding activity of Tau by protein phosphatase 2A. Neuron. 17:1201-1207.

27. Ahlijanian, M.K., et al. 2000. Hyperphosphorylated tau and neurofilament and cytoskeletal disruptions in mice overexpressing human p25, an activator of cdk5. Proc. Natl. Acad. Sci. U. S. A. 97:2910-2915.

28. Augustinack, J.C., Schneider, A., Mandelkow, E.M., and Hyman, B.T. 2002. Specific tau phosphorylation sites correlate with severity of neuronal cytopathology in Alzheimer's disease. Acta Neuropathol. (Berl.) 103:26-35.
29. Cruz, J.C., Tseng, H.C., Goldman, J.A., Shih, H., and Tsai, L.H. 2003. Aberrant Cdk5 activation by $\mathrm{p} 25$ triggers pathological events leading to neurodegeneration and neurofibrillary tangles. Neuron. 40:471-483.

30. Kins, S., et al. 2001. Reduced protein phosphatase $2 \mathrm{~A}$ activity induces hyperphosphorylation and altered compartmentalization of tau in transgenic mice. J. Biol. Chem. 276:38193-38200.

31. Petrucelli, L., et al. 2004. CHIP and Hsp70 regulate tau ubiquitination, degradation and aggregation. Hum. Mol. Genet. 13:703-714.

32. Kosik, K.S., and Shimura, H. 2005. Phosphorylated tau and the neurodegenerative foldopathies. Biochim. Biophys. Acta. 1739:298-310.

33. Dickey, C.A., et al. 2007. The high-affinity HSP90CHIP complex recognizes and selectively degrades phosphorylated tau client proteins. J. Clin. Invest. 117:648-658.

34. Poppek, D., et al. 2006. Phosphorylation inhibits turnover of the tau protein by the proteasome: influence of RCAN1 and oxidative stress. Biochem.J. 400:511-520.

35. Lu, K.P., Hanes, S.D., and Hunter, T. 1996. A human peptidyl-prolyl isomerase essential for regulation of mitosis. Nature. 380:544-547.

36. Yaffe, M.B., et al. 1997. Sequence-specific and phosphorylation-dependent proline isomerization: A potential mitotic regulatory mechanism. Science. 278:1957-1960.

37. Lu, P.J., Zhou, X.Z., Shen, M., and Lu, K.P. 1999. A function of WW domains as phosphoserineor phosphothreonine-binding modules. Science. 283:1325-1328.

38. Zhou, X.Z., Lu, P.J., Wulf, G., and Lu, K.P. 1999. Phosphorylation-dependent prolyl isomerization: A novel signaling regulatory mechanism. Cell. Mol. Life Sci. 56:788-806

39. Pastorino, L., et al. 2006. The prolyl isomerase Pin 1 regulates amyloid precursor protein processing and amyloid-beta production. Nature. 440:528-534.

40. Lu, K.P. 2004. Pinning down cell signaling, cancer, and Alzheimer's disease. Trends Biochem. Sci. 29:200-209.

41. Wulf, G., Finn, G., Suizu, F., and Lu, K.P. 2005. Phosphorylation-specific prolyl isomerization: Is there an underlying theme? Nature Cell Biol. 7:435-441.

42. Lu, K.P., and Zhou, X.Z. 2007. The prolyl isomerase Pin1: a pivotal new twist in phosphorylation signalling and human disease. Nat. Rev. Mol. Cell Biol. 8:904-916.

43. Shen, Z.J., Esnault, S., and Malter, J.S. 2005. The peptidyl-prolyl isomerase Pin 1 regulates the stability of granulocyte-macrophage colony-stimulating factor mRNA in activated eosinophils. Nat. Immunol. 6:1280-1287.

44. Suizu, F., Ryo, A., Wulf, G., Lim, J., and Lu, K.P. 2006. Pin 1 regulates centrosome duplication and its overexpression induces centrosome amplification, chromosome instability and oncogenesis. Mol. Cell. Biol. 26:1463-1479.

45. Butterfield, D.A., et al. 2006. Pin1 in Alzheimer's disease. J. Neurochem. 98:1697-1706.

46. Lu, P.J., Wulf, G., Zhou, X.Z., Davies, P., and Lu, K.P. 1999. The prolyl isomerase Pin1 restores the function of Alzheimer-associated phosphorylated tau protein. Nature. 399:784-788.

47. Zhou, X.Z., et al. 2000. Pin1-dependent prolyl isomerization regulates dephosphorylation of $\mathrm{Cdc} 25 \mathrm{C}$ and tau proteins. Mol. Cell. 6:873-883.

48. Liou, Y.-C., et al. 2003. Role of the prolyl isomerase Pin 1 in protecting against age-dependent neurodegeneration. Nature. 424:556-561.

49. Hamdane, M., et al. 2006. Pin1 allows for differential Tau dephosphorylation in neuronal cells. Mol. Cell. Neurosci. 32:155-160.

50. Sultana, R., et al. 2006. Oxidative modification and down-regulation of Pin 1 in Alzheimer's disease hippocampus: A redox proteomics analysis. Neurobiol. Aging. 27:918-925.
51. Segat, L., et al. 2007. Pin1 promoter polymorphisms are associated with Alzheimer's disease. Neurobiol. Aging. 28:69-74.

52. Wijsman, E.M., et al. 2004. Evidence for a novel late-onset Alzheimer disease locus on chromosome 19p13.2. Am. J. Hum. Genet. 75:398-409.

53. Ryo, A., et al. 2005. Stable suppression of tumorigenicity by Pin 1-targeted RNA interference in prostate cancer. Clin. Cancer Res. 11:7523-7531.

54. Lee, T.H., Perrem, K., Harper, J.W., Lu, K.P., and Zhou, X.Z. 2006. F-box protein Fbx4 targets Pin2/TRF1 for ubiquitin-mediated degradation and regulates telomere maintenance. J. Biol. Chem. 281:759-768.

55. Wulf, G.M., Liou, Y.C., Ryo, A., Lee, S.W., and Lu, K.P. 2002. Role of Pin 1 in the regulation of p 53 stability and 21 transactivation, and cell cycle checkpoints in response to DNA damage. J. Biol. Chem. 277:47976-47979.

56. Hong, M., et al. 1998. Mutation-specific functional impairments in distinct tau isoforms of hereditary FTDP-17. Science. 282:1914-1917.

57. Zhang, B., et al. 2004. Retarded axonal transport of R406W mutant tau in transgenic mice with a neurodegenerative tauopathy. J. Neurosci. 24:4657-4667.

58. Yoshiyama, Y., et al. 2007. Synapse loss and microglial activation precede tangles in a P301S tauopathy mouse model. Neuron. 53:337-351.

59. Caroni, P. 1997. Overexpression of growth-associated proteins in the neurons of adult transgenic mice. J. Neurosci. Methods. 71:3-9.

60. Lu, K.P., Finn, G., Lee, T.H., and Nicholson, L.K. 2007. Prolyl cis-trans isomerization as a molecular timer. Nat. Chem. Biol. 3:619-629.

61. Orlicky, S., Tang, X., Willems, A., Tyers, M., and Sicheri, F. 2003. Structural basis for phosphodependent substrate selection and orientation by the SCFCdc4 ubiquitin ligase. Cell. 112:243-256.

62. Ramelot, T.A., and Nicholson, L.K. 2001. Phosphorylation-induced structural changes in the amyloid precursor protein cytoplasmic tail detected by NMR. J. Mol. Biol. 307:871-884.

63. Jicha, G.A., et al. 1997. A conformation- and phosphorylation-dependent antibody recognizing the paired helical filaments of Alzheimer's disease. J. Neurochem. 69:2087-2095.

64. Passegue, E., Wagner, E.F., and Weissman, I.L. 2004. JunB deficiency leads to a myeloproliferative disorder arising from hematopoietic stem cells. Cell. 119:431-443.

65. Brewer, G.J., Torricelli, J.R., Evege, E.K., and Price, P.J. 1993. Optimized survival of hippocampal neurons in B27-supplemented Neurobasal, a new serum-free medium combination. J. Neurosci. Res. 35:567-576.

66. Ryo, A., et al. 2003. Regulation of NF-kappaB signaling by Pin1-dependent prolyl isomerization and ubiquitin-mediated proteolysis of p65/RelA. Mol. Cell. 12:1413-1426.

67. Moechars, D., et al. 1996. Expression in brain of amyloid precursor protein mutated in the alpha-secretase site causes disturbed behavior, neuronal degeneration and premature death in transgenic mice. EMBO J. 15:1265-1274.

68. Liou, Y.C., et al. 2002. Loss of Pin 1 function in the mouse causes phenotypes resembling cyclin D1-null phenotypes. Proc. Natl. Acad. Sci. U. S. A. 99:1335-1340.

69. Wulf, G., Garg, P., Liou, Y.C., Iglehart, D., and Lu, K.P. 2004. Modeling breast cancer in vivo and ex vivo reveals an essential role of Pin 1 in tumorigenesis. EMBO J. 23:3397-3407.

70. Tozuka, Y., Fukuda, S., Namba, T., Seki, T., and Hisatsune, T. 2005. GABAergic excitation promotes neuronal differentiation in adult hippocampal progenitor cells. Neuron. 47:803-815.

71. Litchfield, S., and Nagy, Z. 2001. New temperature modification makes the Bielschowsky silver stain reproducible. Acta Neuropathol. (Berl.) 101:17-21. 